\title{
NLRP3 tyrosine phosphorylation is controlled by protein tyrosine phosphatase PTPN22
}

\author{
Marianne R. Spalinger, ${ }^{1}$ Stephanie Kasper, ${ }^{1}$ Claudia Gottier, ${ }^{1}$ Silvia Lang, ${ }^{1}$ Kirstin Atrott, ${ }^{1}$ Stephan R. Vavricka, ${ }^{1,2}$ Sylvie Scharl, ${ }^{1}$ \\ Petrus M. Gutte, ${ }^{3}$ Markus G. Grütter, ${ }^{3}$ Hans-Dietmar Beer, ${ }^{4}$ Emmanuel Contassot, ${ }^{4}$ Andrew C. Chan, ${ }^{5}$ Xuezhi Dai, ${ }^{6}$ \\ David J. Rawlings, ${ }^{6}$ Florian Mair, ${ }^{7}$ Burkhard Becher, ${ }^{7}$ Werner Falk, ${ }^{8}$ Michael Fried, ${ }^{1,2}$ Gerhard Rogler, ${ }^{1,2}$ and Michael Scharl ${ }^{1,2}$ \\ 'Division of Gastroenterology and Hepatology, University Hospital Zurich, Zurich, Switzerland. ${ }^{2}$ Zurich Center for Integrative Human Physiology and ${ }^{3}$ Institute of Biochemistry, \\ University of Zurich, Zurich, Switzerland. ${ }^{4}$ Clinic for Dermatology, University Hospital Zurich, Zurich, Switzerland. ${ }^{5}$ Department of Immunology, Genentech, South San Francisco, California, USA. \\ ${ }^{6}$ Seattle Children's Research Institute, Division of Immunology, Seattle, Washington, USA. 'Institute of Experimental Immunology, University of Zurich, Zurich, Switzerland. \\ ${ }^{8}$ Department of Internal Medicine I, University Hospital Regensburg, Regensburg, Germany.
}

\begin{abstract}
Inflammasomes form as the result of the intracellular presence of danger-associated molecular patterns and mediate the release of active IL-1 $\beta$, which influences a variety of inflammatory responses. Excessive inflammasome activation results in severe inflammatory conditions, but physiological IL-1 $\beta$ secretion is necessary for intestinal homeostasis. Here, we have described a mechanism of NLRP3 inflammasome regulation by tyrosine phosphorylation of NLRP3 at Tyr861. We demonstrated that protein tyrosine phosphatase non-receptor 22 (PTPN22), variants in which are associated with chronic inflammatory disorders, dephosphorylates NLRP3 upon inflammasome induction, allowing efficient NLRP3 activation and subsequent IL-1 $\beta$ release. In murine models, PTPN22 deficiency resulted in pronounced colitis, increased NLRP3 phosphorylation, but reduced levels of mature IL-1ß. Conversely, patients with inflammatory bowel disease (IBD) that carried an autoimmunity-associated PTPN22 variant had increased IL-1 $\beta$ levels. Together, our results identify tyrosine phosphorylation as an important regulatory mechanism for NLRP3 that prevents aberrant inflammasome activation.
\end{abstract}

\section{Introduction}

Inflammasomes are large, multiprotein complexes that form upon cytosolic or nuclear presence of damage- or pathogen-associated molecular patterns, ultimately leading to the secretion of IL-1 $\beta$ and IL-18. Inflammasomes consist of a danger-sensing receptor, the adaptor molecule ASC, and the protease caspase-1. Assembly of the inflammasome complex results in autocleavage and activation of caspase-1, which cleaves pro-IL-1 $\beta$ and pro-IL-18 into their active forms and mediates their secretion (1). Several NOD-like receptor (NLR) proteins, including NLRP3, NLRP1, NLRC4, and absent in melanoma 2 (AIM2), are able to form inflammasomes and induce subsequent IL-1 $\beta$ secretion (2). All these receptors have similar structures, containing an $\mathrm{N}$-terminal caspase recruitment and activation domain (CARD) or a pyrin domain (PYD) that mediates interaction with downstream proteins; a nucleotide oligomerization domain (NOD; not present in AIM2) that allows oligomerization upon activation; and one or several leucine-rich repeats (LRRs) or, in the case of AIM2, a HIN domain, which are involved in ligand binding and activation of the receptor. Upon activation, inflammasome receptors containing a CARD directly recruit caspase-1, while inflammasome receptors containing a PYD rely on the adaptor molecule ASC for caspase-1 recruitment and activation. Each inflammasome-associated danger-sensing receptor is activated by specific ligands, including the NLRC4 ligand flagellin; bacterial

Authorship note: G. Rogler and M. Scharl contributed equally to this work Conflict of interest: A.C. Chan is an employee of Genentech.

Submitted: July 15, 2015; Accepted: February 24, 2016.

Reference information: / Clin Invest. 2016;126(5):1783-1800. doi:10.1172/JCI83669. products in the case of NLRP1; or double-stranded DNA (dsDNA) that activates AIM2. NLRP3 is activated by a variety of different molecules, including muramyl dipeptide (MDP), titanium dioxide $\left(\mathrm{TiO}_{2}\right)$, silicium dioxide $\left(\mathrm{SiO}_{2}\right)$, monosodium urate crystals (MSUs), and extracellular ATP (3). The mechanism whereby NLRP3 is activated by this variety of compounds is still not understood, but production of ROS and potassium efflux have been described as mediators leading to NLRP3 activation. Recent reports demonstrated that tyrosine phosphorylation of ASC acts as a molecular switch for activation of inflammasome assembly (4), and serine phosphorylation of NLRC4 has been shown to be important for its activation (5). This demonstrates that the phosphorylation status of certain proteins in the inflammasome complex influence inflammasome activity.

Deregulated NLRP3 activation is detrimental for the host, and several immune disorders have been associated with aberrant NLRP3 activation, including Crohn's disease (CD) (6), systemic lupus erythematosus (SLE), rheumatoid arthritis (RA), and type 1 diabetes (T1D) $(3,7)$. In most cases, increased NLRP3 activation results in uncontrolled inflammation that drives disease progression (3), but in intestinal inflammation, NLRP3 activation seems to play a dual role. While exacerbated IL-1 $\beta /$ IL-18 secretion is observed in active lesions from patients with inflammatory bowel diseases (IBDs) (8), and NLRP3-deficient animals have been shown to have less severe experimental colitis in some reports (9), other groups reported defective epithelial barrier function and increased intestinal inflammation in NLRP3-deficient animals (10). Further, mice deficient for the inflammasome adaptor molecule ASC or for caspase- 1 have epithelial barrier defects and increased intestinal inflammation, and it has been 



B

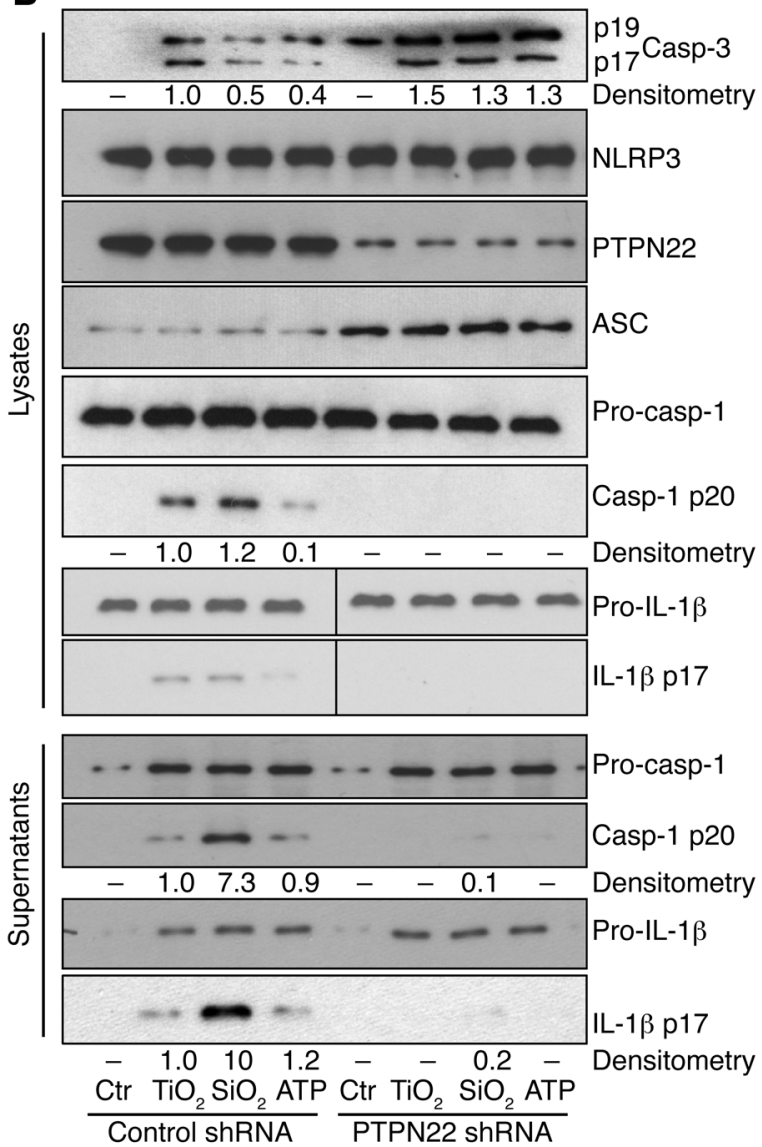

Figure 1. Knockdown of PTPN22 reduces IL-1 $\beta$ secretion. PTPN22 knockdown was induced in THP-1 cells using lentiviral shRNA expression vectors. Cells were pretreated for 12 hours with upLPS before activation with MDP (100 ng/ml, 24 hours), MSU ( $150 \mathrm{ng} / \mathrm{ml}, 6$ hours), $\mathrm{TiO}_{2}$ (150 $\mathrm{ng} / \mathrm{ml}, 24$ hours), $\mathrm{SiO}_{2}(150 \mathrm{ng} /$ $\mathrm{ml}, 12$ hours), or ATP (200 mM, 30 minutes). (A) Cell culture supernatants were analyzed for IL-1 $\beta$ secretion, LDH release, and IL-6 secretion by ELISA. (B) Cell lysates or supernatants were analyzed for caspase-1 (Casp-1), caspase-3 (Casp-3), IL-1 $\beta$, NLRP3, ASC, and PTPN22 expression. Blots for $\mathrm{IL}-1 \beta$ were run on the same gel but were discontinuous. Data are representative of 1 of at least 3 independent experiments with 3-5 replicas $\left(n=3-5 ;{ }^{*} P<0.05\right.$, ${ }^{* *} P<0.01$; Newman-Keuls post hoc test). Numbers below the blots show results of densitometry (cleaved forms). suggested that inflammasome activation mediates intestinal wound healing and epithelial cell reconstitution (11).

Variations within the gene locus encoding protein tyrosine phosphatase non-receptor 22 (PTPN22) are associated with several inflammatory disorders, including CD (12), RA (13), SLE (14), and T1D (15), diseases linked with altered inflammasome function $(6,16)$. Of note, a variant that results in in vitro gain-offunction phosphatase activity (17) in PTPN22 is associated with decreased risk for $\operatorname{CD}(12,18,19)$ while resulting in enhanced susceptibility to RA (20) and SLE (14). We previously demonstrated that PTPN22 expression is reduced in patients with IBD (21), and knockdown of PTPN22 results in increased secretion of proinflammatory cytokines including IL-6 and IL-8 (21, 22). Further, PTPN22 is involved in autophagy activation and MDP-induced signaling and cytokine secretion (22). A study by Wang et al. showed that knockout of PTPN22 results in enhanced susceptibility to experimental arthritis as well as DSS-induced colitis (23). However, a functional role for PTPN22 in inflammasome activation and IL-1 $\beta$ secretion has not been addressed so far.

Here we report what we believe to be a novel regulatory mechanism of NLRP3 inflammasome activation, mediated by tyrosine phosphorylation of NLRP3 on Tyr861. We found that PTPN22 interacts with and dephosphorylates NLRP3 upon proinflammatory insults, allowing robust NLRP3 activation and IL-1 $\beta$ secretion. In PTPN22-deficient cells and mice, NLRP3 phosphorylation was enhanced and inflammasome assembly reduced, resulting in decreased IL-1 $\beta$ secretion.

\section{Results}

Loss of PTPN22 reduces mature $I L-1 \beta$ secretion. To assess a possible effect of PTPN22 on inflammasome activation, we first tested whether loss of PTPN22 influences IL-1 $\beta$ secretion. Knockdown of PTPN22 was induced in THP-1 cells by transfection with a lentiviral vector encoding either nonrelevant control or PTPN22-specific shRNA. After selection of stable shRNA-expressing clones, the cells were differentiated for 48 hours with PMA; pretreated for 12 hours with ultrapure LPS (upLPS); and activated with the NLRP3 inflammasome activators MDP (100 ng/ml, 24 hours), MSU (150 $\mu \mathrm{g} / \mathrm{ml}, 6$ hours $), \mathrm{SiO}_{2}(150 \mu \mathrm{g} / \mathrm{ml}, 6$ hours $), \mathrm{TiO}_{2}(150 \mu \mathrm{g} / \mathrm{ml}$, 6 hours), and ATP ( $2 \mathrm{mM}, 30$ minutes). In response to all treatments, PTPN22 knockdown resulted in a significant reduction of agonist-induced IL-1 $\beta$ release (Figure 1A). Since inflammasome activation induces pyroptosis, an inflammation-associated form of cell death, reduction of inflammasome activity is expected to result in reduced cell lysis. Despite decreased inflammasome activation, release of lactate dehydrogenase (LDH), a general marker of cell damage, was not affected by loss of PTPN22 (Figure 1A). Therefore, we hypothesized that PTPN22 might induce another form of cell death. To address possible mechanisms, we analyzed the cells for the apoptosis marker caspase-3. Since there was an increase in levels of cleaved caspase-3 (Figure 1B), we suggest that loss of PTPN22 might promote apoptosis, but more detailed analyses would be necessary to draw a final conclusion. Since secretion of other inflammatory cytokines, such as IL-6 or TNF, was enhanced or remained unchanged by loss of PTPN22 (Figure 
A

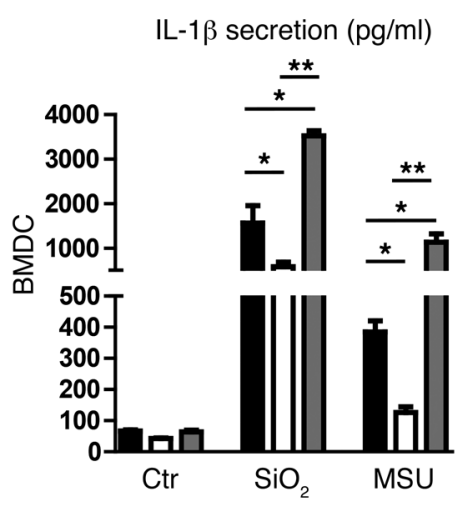

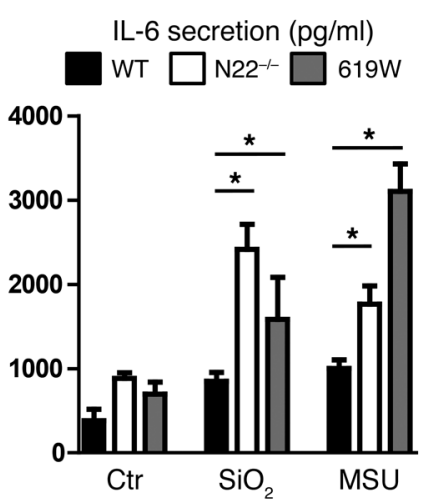

IL-6 secretion $(\mathrm{pg} / \mathrm{ml})$
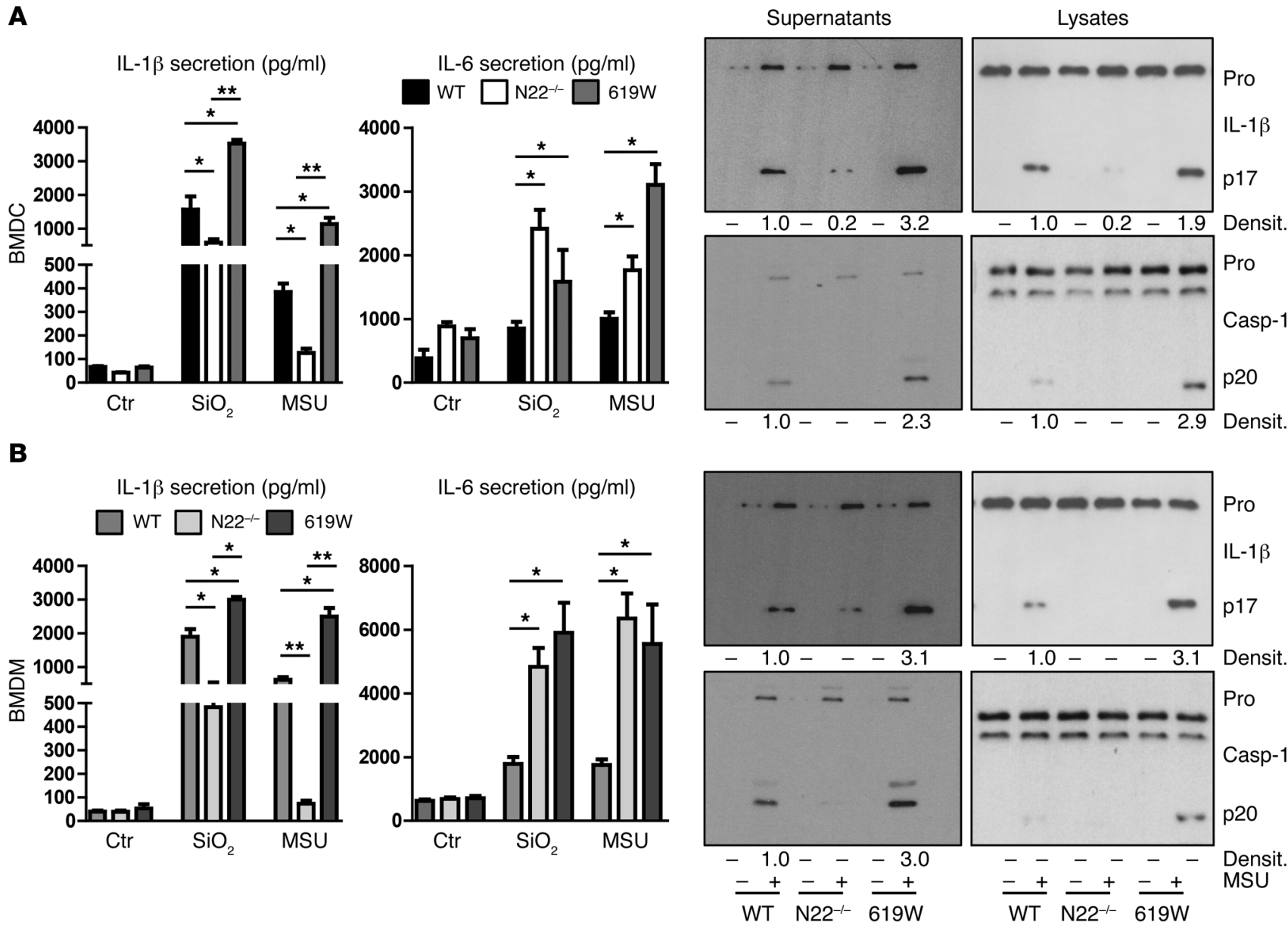

Figure 2. Loss of PTPN22 reduces and presence of the PTPN22-619W variant enhances IL-1 $\beta$ secretion. (A) BMDCs and (B) BMDMs were derived from Ptpn22-/- (N22---) and WT littermates, or mice expressing the autoimmunity-associated PTPN22 variant (619W), pretreated for 12 hours with upLPS prior to activation with MSU or $\mathrm{SiO}_{2}$, and analyzed for IL-1 $\beta$ and IL- 6 release by ELISA, as well as caspase-1/IL-1 $1 \beta$ cleavage by Western blot. Data are representative of 1 of at least 3 independent experiments with 3-5 replicas $\left(n=3-5 ;{ }^{*} P<0.05,{ }^{* *} P<0.01\right.$; Newman-Keuls post hoc test). Numbers below the blots show results of densitometry (cleaved forms). Ctr, control.

1A and Supplemental Figure 1A; supplemental material available online with this article; doi:10.1172/JCI83669DS1), the decrease in IL-1 $\beta$ secretion seemed to result not merely from changes in cell death. To determine whether the observed reduction in IL-1 $1 \beta$ was due to reduced levels of pro- or cleaved IL-1 $\beta$, we subjected cell culture supernatants and cell lysates to Western blot analysis. While cell damage-mediated release of pro-IL-1 $\beta$ and pro-caspase-1 was not changed upon loss of PTPN22, levels of mature IL-1 $\beta$, as well as of cleaved caspase-1, were reduced (Figure 1B). The reduction in mature IL-1 $\beta$ was more pronounced in the Western blot analysis compared with the ELISA measurement (Figure $1, A$ and $B)$. This is because IL-1 $\beta$ ELISA detects not only mature IL- $1 \beta$ but also pro-IL-1 $\beta$, and the dynamic range of the ELISA is several magnitudes higher than that of the Western blot. Similar IL- $1 \beta$ secretion results were obtained in $\mathrm{SiO}_{2}$ - and MSU-treated human MM6 monocytic cells (Supplemental Figure 1B) and in ultraviolet light-treated (UV-treated) primary human keratinocytes transfected with either control or PTPN22-targeting siRNA (Supplemental Figure 1C; note that keratinocytes express PTN22, although they are not of hematopoietic origin), as well as in primary bone marrow-derived dendritic cells (BMDCs) and bone marrow-derived macrophages (BMDMs) from PTPN22-knockout (Ptpn22--) mice ex vivo: BMDCs and BMDMs derived from Ptpn $22^{--}$mice secreted less mature IL-1 $\beta$ in response to $\mathrm{SiO}_{2}$ or MSU than BMDCs/BMDMs from WT mice, while IL-6 secretion was enhanced and TNF secretion remained unchanged (Figure 2, A and B, and Supplemental Figure 2A). Again, LDH release was similar in the two genotypes (Supplemental Figure 2A), while levels of cleaved caspase- 1 and mature IL-1 $\beta$ were lower in BMDCs/ BMDMs from Ptpn22- animals than in those from WT animals treated with either $\mathrm{SiO}_{2}$ or MSU (Figure 2, A and B). On the other hand, BMDCs/BMDMs from mice expressing the autoimmunity-associated variant in PTPN22 (619W mice) showed increased levels of cleaved caspase-1/IL-1 $\beta$ and enhanced IL-1 $\beta$ secretion (Figure 2). Of note, the autoimmunity-associated variant was initially described to result in a gain-of-function protein (17), although some newer studies indicate an in vivo loss-of-function effect in $\mathrm{T}$ cell biology (24) and altered effects in T lymphocyte, B lymphocyte, and dendritic cell biology (25). Of note, Western blot analysis of cell lysates did not reveal changes in pro-IL-1 $\beta$, NLRP3, or pro-caspase-1 protein levels, while ASC levels were enhanced in PTPN22-deficient cells (Figure 1B and Figure 2). 
A
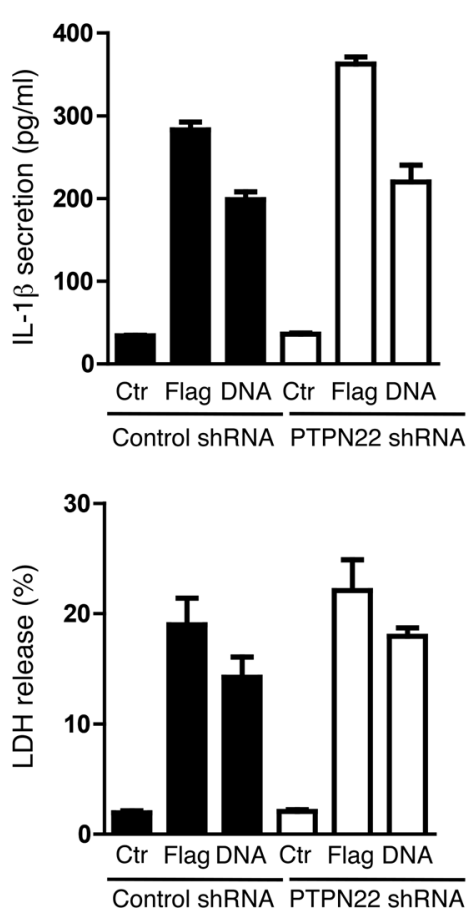

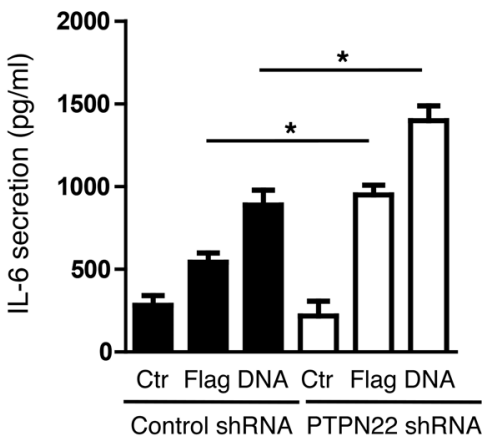

$\mathbf{B}$

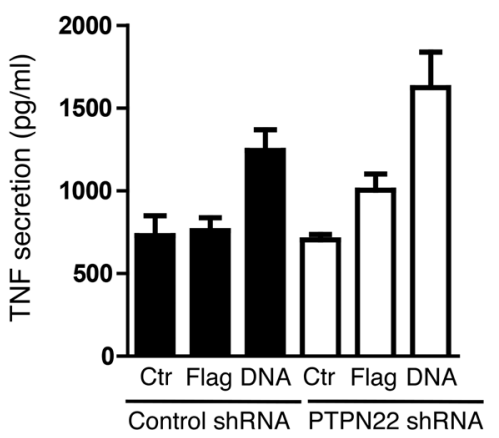

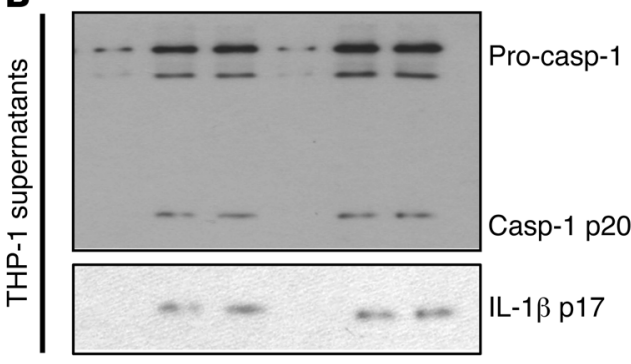

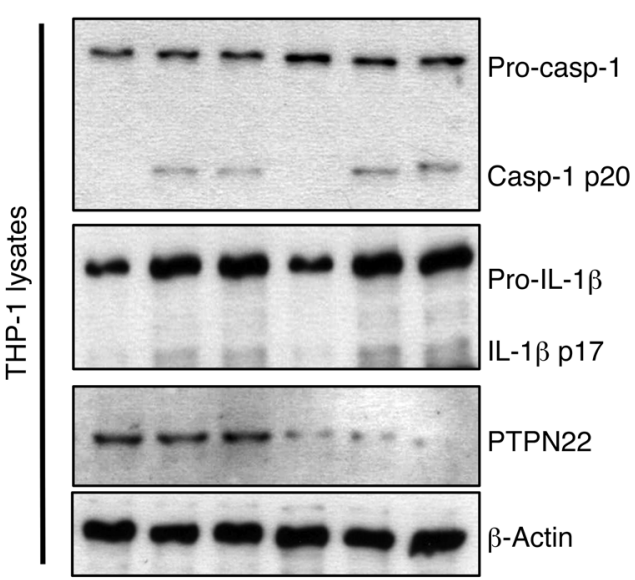

Figure 3. PTPN22 does not affect NLRC4- or AIM2-mediated IL-13 secretion. THP-1 cells expressing control or PTPN22-targeting shRNA were pretreated for 12 hours with upLPS before transfection with flagellin (Flag) or dsDNA and analyzed for (A) IL-1 13, IL-6, LDH, and TNF release by ELISA and (B) caspase-1 cleavage and IL-1 $\beta$ maturation by Western blot. Data are representative of 4 independent experiments. ${ }^{*} P<0.05$, Newman-Keuls post hoc test.

To confirm that the reduction in cleaved IL-1 $\beta$ affects bioactive IL-1, we cultured D10.G4.1 cells, which respond to the presence of bioactive IL-1 with proliferation, in the presence of cell culture supernatant from MSU-treated THP-1 cells or BMDCs. In the presence of supernatant from MSU-treated WT BMDCs or THP-1 cells expressing control shRNA, the cells proliferated, and this was blocked upon addition of an IL-1 receptor antagonist (Supplemental Figure 2B and data not shown). With supernatants from Ptpn22-- BMDCs or THP-1 cells expressing PTPN22specific shRNA, proliferation was reduced (Supplemental Figure $2 B)$, indicating that differences in IL-1 $\beta$ measurements in ELISA and Western blot analysis are specific for bioactive IL-1 $\beta$. Further, this demonstrates that the reduction in IL-1 $\beta$ upon loss of PTPN22 has profound consequences for the proliferation of IL-1-dependent cells, suggesting the physiological relevance of our findings.

In MDP-treated THP-1 cells, loss of PTPN22 resulted in reduced expression of pro-IL1B mRNA after 24 hours, likely due to the effect of PTPN22 on MDP-induced signaling in monocytic cells (22), while in MM6 cells and BMDCs, loss of PTPN22 did not affect MDP-induced $I L 1 B / I l 1 b$ or NLRP3/Nlrp3 mRNA expression (Supplemental Figure 2C). As PTPN22 has been implicated in affecting LPS-mediated TLR4 responses (23), we next addressed whether loss or altered function of PTPN22 might affect LPSinduced NLRP3/IL-1 $\beta$ expression. When we used upLPS as a priming agent, loss of PTPN22 had no effect on IL1B or NLRP3 mRNA expression (Supplemental Figure 2C). upLPS efficiently induced IL-1 $\beta$ and NLRP3 mRNA/protein, and there was no difference observed among WT, Ptpn22 $2^{--}$, and 619W BMDCs (Supplemental Figure 2, C-E). BioGPS datasets (26) suggest a reduction in
PTPN22 expression upon LPS treatment, and indeed, after 8 hours of upLPS treatment, Ptpn 22 mRNA levels were reduced in WT BMDCs. However, after 2 hours and 16 hours Ptpn 22 mRNA expression was increased, and PTPN22 protein levels were clearly enhanced upon upLPS treatment at all analyzed time points (Supplemental Figure 2, D and E). Further, and in line with other publications $(23,27,28)$, both loss of PTPN22 and the presence of the autoimmunity-associated variant resulted in decreased induction of type I IFN (Supplemental Figure 2D). Since type I IFNs are involved in caspase-11 induction (29), and caspase-11 is involved in noncanonical IL-1 $\beta$ cleavage/secretion upon LPS treatment (29, 30), we further addressed expression/cleavage of caspase-11. In our hands, upLPS robustly induced expression and cleavage of caspase-11, but there was no difference among PTPN22-deficient, PTPN22-WT, and PTPN22-619W-expressing cells (Supplemental Figure $2 \mathrm{E}$ ), ruling out that the observed effects on IL- $1 \beta$ secretion might result from changes in caspase-11 activity. Taken together, our data indicate that loss of PTPN22 results in a reduction in mature IL-1 $\beta$ release, without reducing the expression of pro-IL-1 $\beta$ or other inflammasome components.

NLRC4- and AIM2-mediated IL-1 $\beta$ secretion is not affected upon loss of PTPN22. As other pattern recognition receptors (PRRs) besides NLRP3 can form inflammasome complexes, we next addressed whether PTPN22 is also involved in mature IL-1 $\beta$ secretion upon activation of the NLRC4 or AIM2 inflammasome. Flagellin (a NLRC4 ligand; ref. 5) and dsDNA (inducing AIM2 activation; ref. 31) transfection of THP-1 cells resulted in IL-1 $\beta$ secretion, without a significant difference between PTPN22-expressing and PTPN22-deficient cells. Also, LDH release and TNF secretion 
A

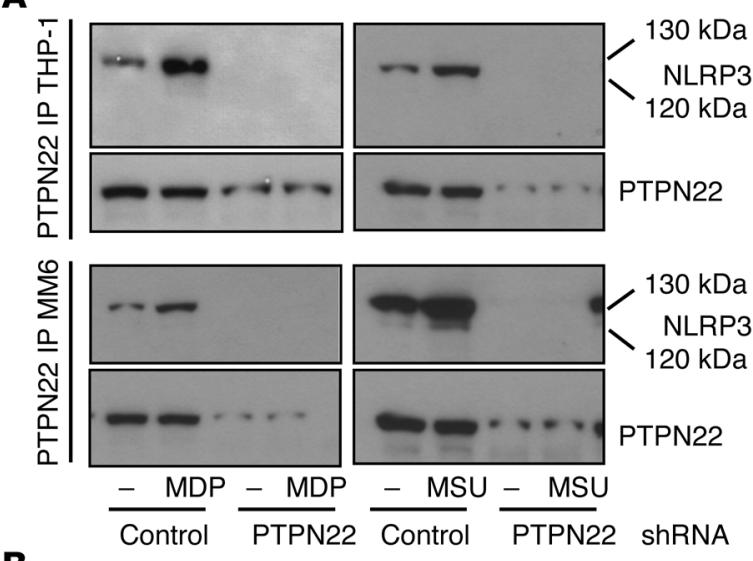

B

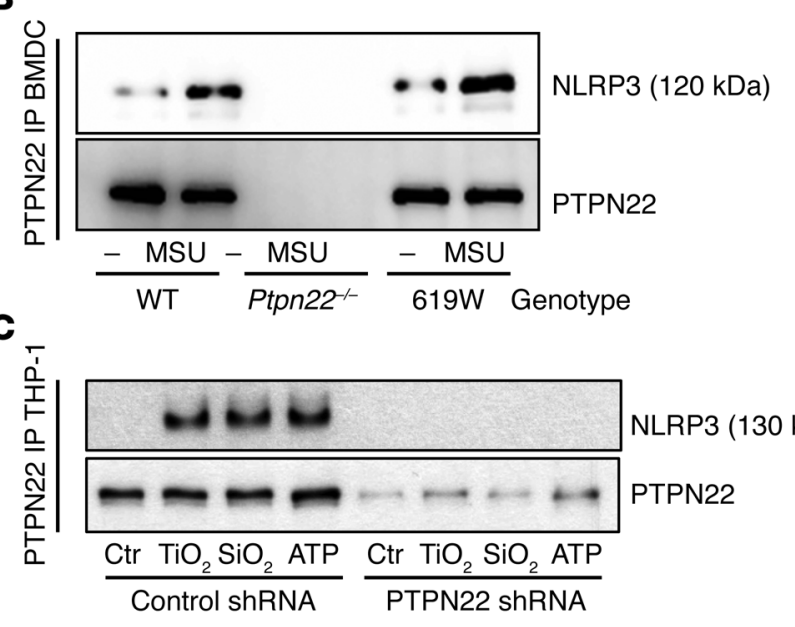

D
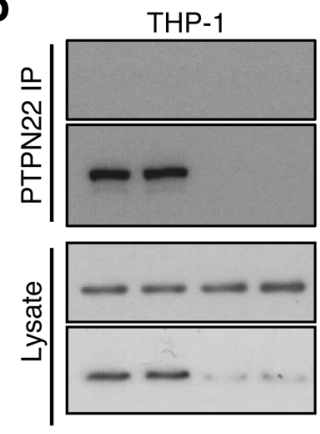

- dsDNA - dsDNA

$\overline{\text { Control }} \overline{\text { PTPN22 }}$

E
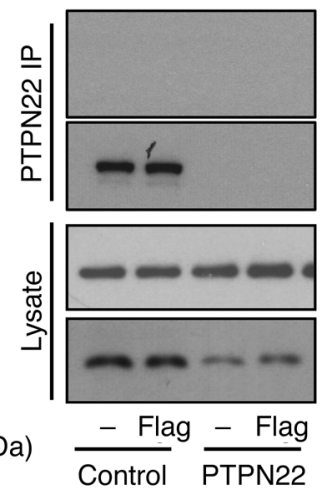

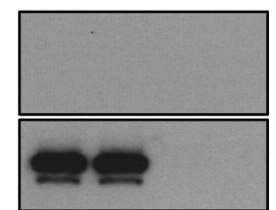

NLRC4

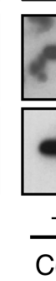

BMDC

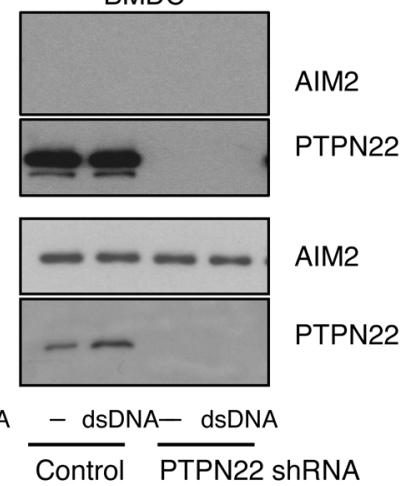

PTPN22

NLRC4

PTPN22

$\frac{- \text { Flag }}{\text { Control }} \frac{- \text { Flag }}{\text { PTPN22 }}$ shRNA

Figure 4. PTPN22 interacts with NLRP3 upon inflammasome activation. THP-1 cells, MM6 cells, and BMDCs were treated with upLPS for 12 hours prior to activation with MDP (100 ng/ml, 24 hours), MSU ( $100 \mathrm{ng} / \mathrm{ml}, 6$ hours), $\mathrm{TiO}_{2}\left(150 \mathrm{ng} / \mathrm{ml}, 24\right.$ hours), $\mathrm{SiO}_{2}(150 \mathrm{ng} / \mathrm{ml}, 24$ hours), ATP (2 mM, $30 \mathrm{minutes})$, dsDNA, or flagellin, as indicated. (A and C) PTPN22 was precipitated from THP-1 or MM6 lysates and analyzed by Western blot for coprecipitated NLRP3. (B) PTPN22 was precipitated from BMDCs and analyzed for NLRP3. (D) PTPN22 was precipitated from dsDNA-treated THP-1 cells or BMDCs and analyzed by Western blot for coprecipitation of AIM2. (E) PTPN22 was precipitated from dsDNA-treated THP-1 cells or BMDCs and analyzed by Western blot for coprecipitation of NLRC4. Data are representative of 1 of 3-5 independent experiments.

remained unchanged, while IL-6 was again increased (Figure 3). Correspondingly, loss of PTPN22 did not affect levels of cleaved caspase-1 or mature IL-1 $\beta$ in cell lysates or cell supernatants from THP-1 cells treated with either flagellin or dsDNA (Figure 3). This indicates that PTPN22 specifically modulates NLRP3-mediated inflammasome activation but does not affect activity of NLRC4 or AIM2 inflammasomes.

NLRP3 interacts with PTPN22 and is regulated by tyrosine phosphorylation. Having shown that PTPN22 specifically regulates NLRP3 dependent IL-1 $\beta$ secretion, we next investigated the possible molecular mechanism underlying this effect. We first investigated whether PTPN22 interacts directly with the NLRP3 inflammasome complex. Immunoprecipitation of PTPN22 and subsequent immunoblotting for NLRP3 revealed a direct interaction between PTPN22 and NLRP3 that was enhanced upon MDP or MSU treatment in human THP-1 and MM6 cells, as well as in primary mouse BMDCs (Figure 4, A and B). The same was true for the altered-function PTPN22 (PTPN22-619W) variant in BMDCs. Basal interaction of PTPN22-619W with NLRP3 appeared to be slightly increased when compared with WT PTPN22, and also in these cells, inflammasome activation increased the interac- tion (Figure 4B and Supplemental Figure 3A, left). This was also observed in THP-1 cells treated with other NLRP3 inflammasome activators, namely $\mathrm{TiO}_{2}, \mathrm{SiO}_{2}$, and ATP (Figure 4C). On the other hand, PTPN22 did not interact with NLRC4 or AIM2, regardless of their activation status (Figure 4, D and E).

To confirm this observation, we immunoprecipitated NLRP3 from the respective cell lysates and immunoblotted for PTPN22. Again, upon MDP or MSU treatment, we detected PTPN22 in NLRP3 immunoprecipitates from human THP-1 and MM6 cells, as well as from mouse BMDCs (Figure 5, A and B), while NLRP3 did not interact with the related phosphatase PTPN2 (Figure 5A and Supplemental Figure 3B), indicating a specific interaction between PTPN22 and NLRP3. The direct interaction between NLRP3 and PTPN22 prompted us to address whether a component of the NLRP3 inflammasome might be tyrosine phosphorylated, since PTPN22 regulates its substrates via tyrosine dephosphorylation. Given the direct interaction between NLRP3 and PTPN22, we first probed the NLRP3 precipitates from THP-1 monocytes, MM6 macrophages, or primary mouse BMDCs treated with either MSU or MDP for phosphotyrosine (pTyr). We detected a pTyr band in the precipitates at the height of full- 
A

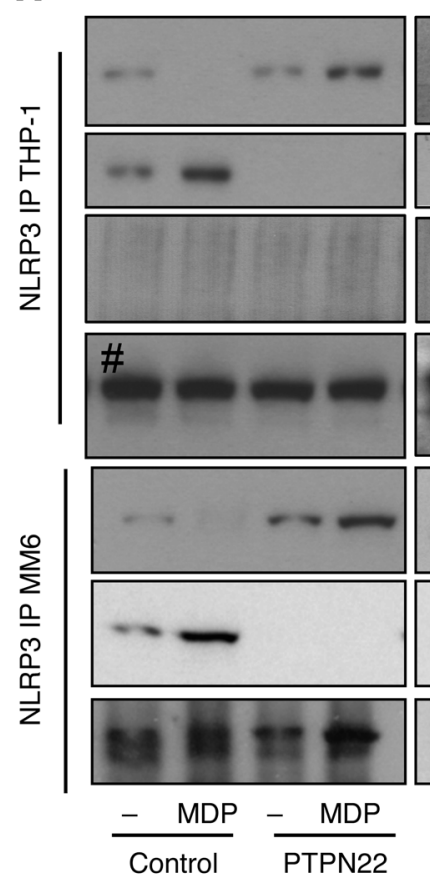

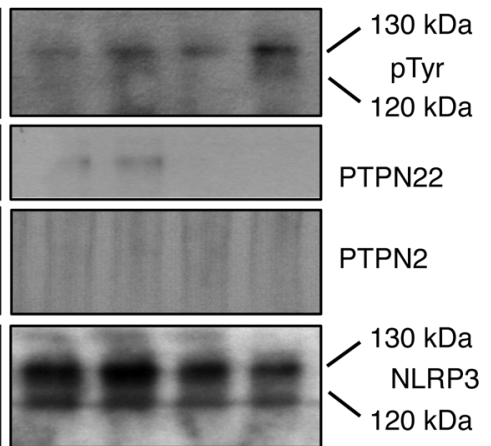

pTyr

$130 \mathrm{kDa}$

PTPN22

$130 \mathrm{kDa}$

NLRP3

$120 \mathrm{kDa}$
B

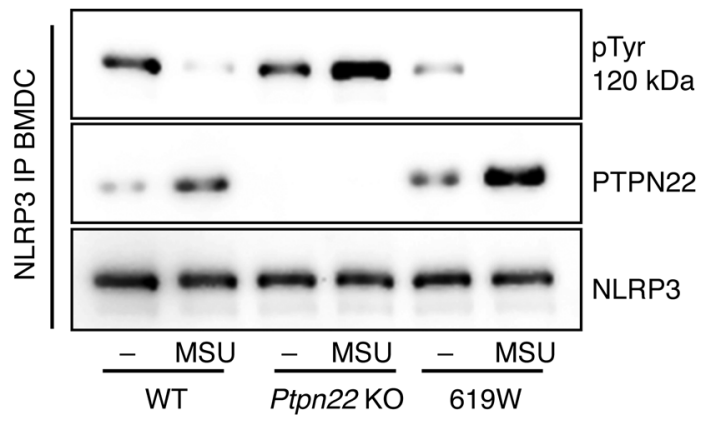

C

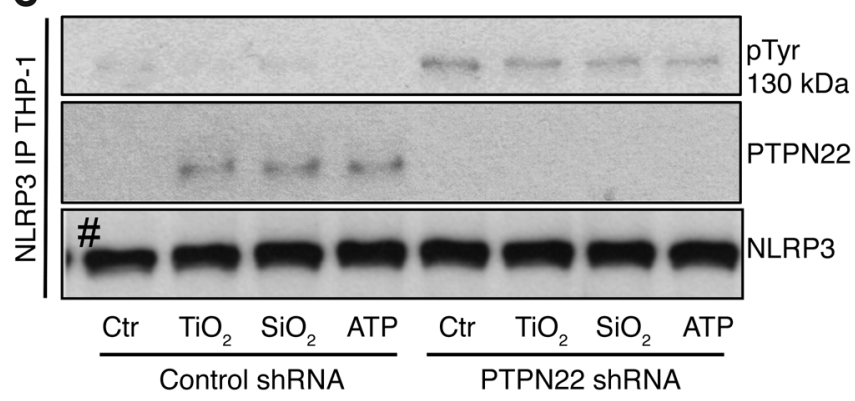

D NLRP3 IP $\frac{-\mathrm{MSU}}{\text { Control }} \frac{-\mathrm{MSU}}{\mathrm{PTPN} 22}$ shRNA PTPN22 IP

E $\mathrm{RTyr}$

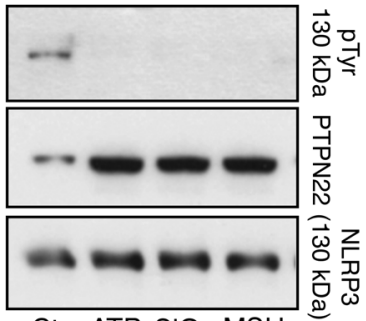

Ctr ATP $\mathrm{SiO}_{2} \mathrm{MSU}$

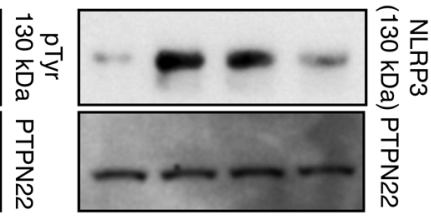

Ctr ATP $\mathrm{SiO}_{2} \mathrm{MSU}$

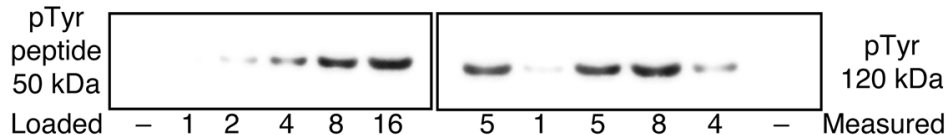

NLRP3

$120 \mathrm{kDa}$

Loaded


NLRP3

$120 \mathrm{kDa}$

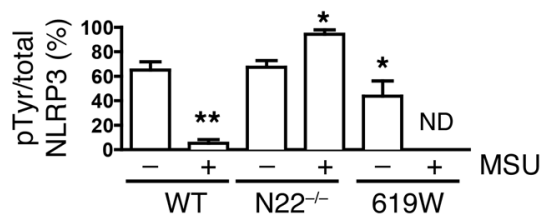

Figure 5. NLRP3 is tyrosine phosphorylated. THP-1 and MM6 cells (A and C), BMDCs (B), and PBMCs (D) were treated with upLPS for 12 hours prior to activation with MDP (100 ng/ml, 24 hours), MSU (100 ng/ml, 6 hours), TiO 2 ( $150 \mathrm{ng} / \mathrm{ml}, 24$ hours), SiO $(150 \mathrm{ng} / \mathrm{ml}, 24$ hours), ATP (2 mM, 30 minutes), dsDNA, or flagellin, as indicated. NLRP3 was precipitated from cell lysates, and precipitates were analyzed for tyrosine phosphorylation, coprecipitated PTPN22, or coprecipitated PTPN2. (D) PTPN22 was precipitated in addition to NLRP3 and analyzed for coprecipitated NLRP3. (E) Amounts of NLRP3 and pTyr in NLRP3 precipitates were quantified using standard curves for NLRP3 and a pTyr peptide. The graph below the representative Western blots shows statistical analysis of densitometry. ND, not detected. Left and right blots in $\mathbf{E}$ were run on the same gel, but were discontinuous. Numbers below the blots in $\mathbf{D}$ show the amount of loaded NLRP3 or pTyr peptide (left) and measured amounts (right), respectively. The pound symbol (\#) marks blots where the $130-\mathrm{kDa}$ and $120-\mathrm{kDa}$ forms of NLRP3 are not completely separated and appear as single bands. Data shown are from 1 of 3-5 independent experiments. ${ }^{*} P<0.05,{ }^{* *} P<0.01$, Student's $t$ test with Bonferroni correction.

length NLRP3 in all of our tested cell types (130 kDa in human cells, $120 \mathrm{kDa}$ in mouse tissue; Figure 5 and Supplemental Figure $3 \mathrm{~A}$ ). Of note, this pTyr band was increased upon loss of PTPN22, while it was reduced with the presence of the PTPN22$619 \mathrm{~W}$ variant (Figure 5B). While in nonactivated cells, PTPN22NLRP3 interaction was low or absent (Figures 4 and 5), activation resulted in increased interaction and efficient dephosphorylation of NLRP3 (Figure 5 and Supplemental Figure 3A). Since it has recently been reported that inflammasome activation is dependent on ASC phosphorylation, we further studied whether loss of PTPN22 additionally affects ASC tyrosine phosphorylation in mouse BMDCs. Precipitation of ASC and subsequent probing for pTyr revealed tyrosine phosphorylation of ASC upon inflammasome activation, but there was no detectable difference between WT and Ptpn22-- cells (Supplemental Figure 4).

In human primary peripheral blood mononuclear cells (PBMCs), we also detected a physical interaction between PTPN22 and NLRP3 and observed tyrosine phosphorylation of NLRP3. Upon NLRP3 inflammasome activation, as measured by IL- $1 \beta$ secretion and cleavage of caspase- 1 and IL- $1 \beta$ (data not shown), NLRP3 tyrosine phosphorylation was decreased (Figure 5D). Using a semiquantitative approach, we found that roughly 60\%-80\% of NLRP3 was tyrosine phosphorylated in nonactivated cells, and the level dropped to below 10\% upon MSU treatment (Figure 5E). Upon loss of PTPN22, basal NLRP3 phosphorylation was not altered, but upon inflammasome activation, phosphoryla- 
A

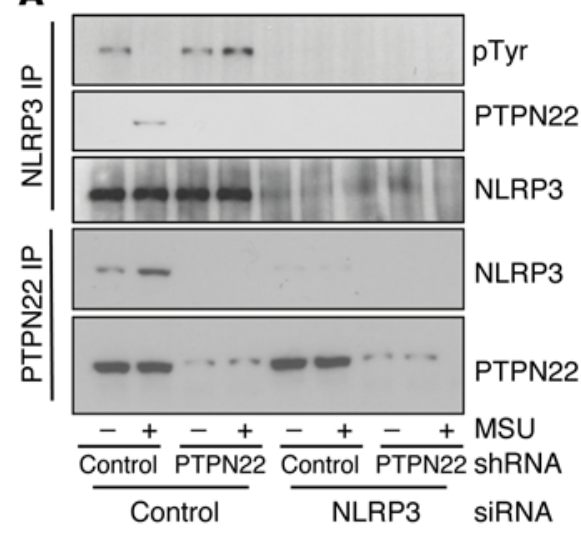

B

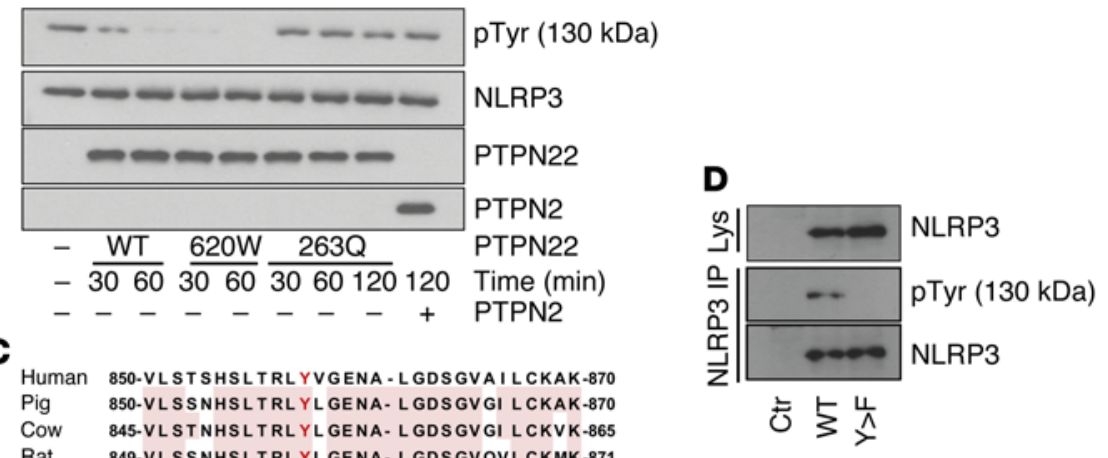

E

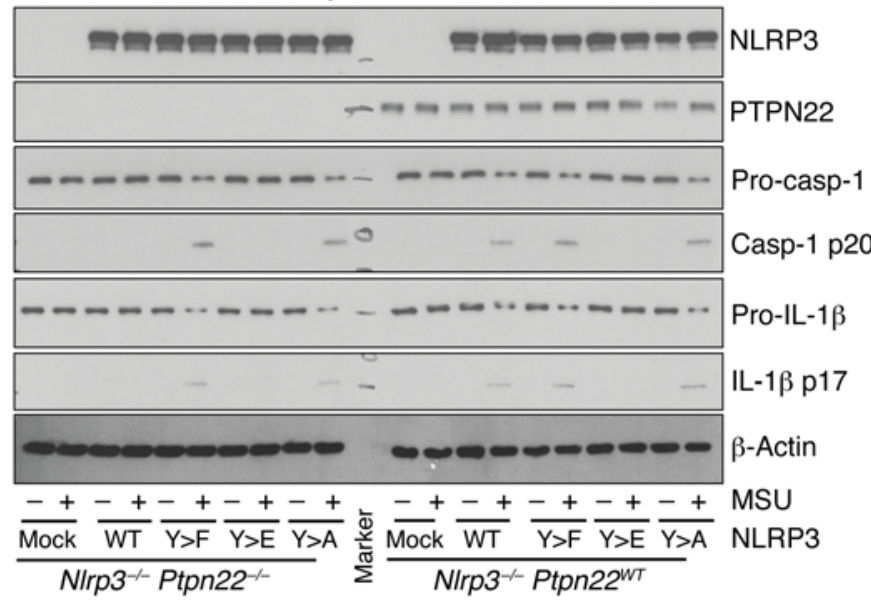

MOuse

Chicken 742-AL GTST CLEELDLSF SAGLRDDGVKLL CGL Q-772
F Supernatant

.

.

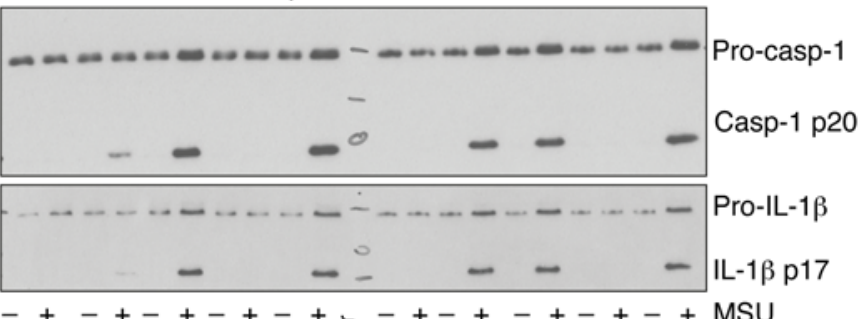

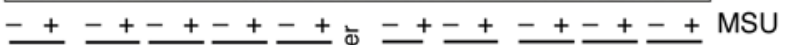

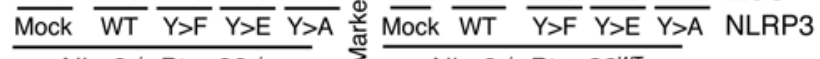

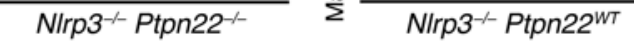

G NLRP3 IP from BMDC

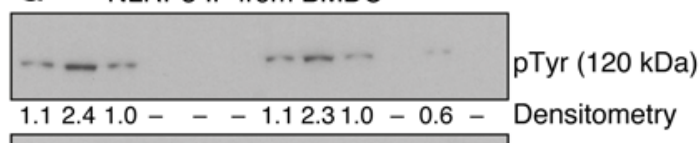

$1.12 .41 .0-\quad-1.12 .31 .0-0.6-$ Densitometry

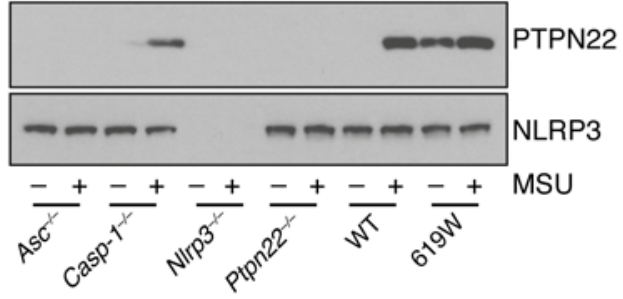

Figure 6. NLRP3 is tyrosine phosphorylated at Tyr861. (A) THP-1 cells expressing control or PTPN22-targeting shRNA were treated with control or NLRP3-targeting siRNA constructs and incubated for 12 hours with upLPS before activation with MSU (6 hours). NLRP3 or PTPN22 was precipitated from the cell lysates and analyzed for tyrosine phosphorylation and PTPN22 coprecipitation or NLRP3 coprecipitation, respectively. (B) Purified NLRP3 was incubated in phosphatase buffer for 60 minutes in the presence or absence of purified WT-PTPN22 (WT), altered-function PTPN22 (619W), loss-of-function PTPN22 (263Q), or purified PTPN2. (C) Multiple sequence alignment of NLRP3 sequences from the indicated species using FASTA.2 (http://www.ebi.ac.uk) reveals conservation of the tyrosine at position 861. (D) HEK293T cells were transfected with WT NLRP3 or a NLRP3 construct where Tyr861 was replaced by a phenylalanine $(\mathrm{Y}>\mathrm{F})$. NLRP3 was immunoprecipitated and analyzed for the presence of pTyr. Lys, lysates. (E and F) Nlrp3 ${ }^{-1-}$ BMDCs were left nontransfected or transfected with WT NLRP3; Y >F NLRP3; or NLRP3 where Tyr858 was replaced with a glutamine to mimic constitutive phosphorylation ( $Y>E$ ) or by an alanine (control to glutamine substitution $[Y>A]$ ) and activated with MSU. Lysates $(E)$ and supernatants (F) were analyzed for the indicated proteins. (G) BMDCs were derived from ASC-, caspase-1-, NLRP3-, or PTPN22-deficient mice or mice expressing the autoimmunity-associated PTPN22 variant (619W); pretreated for 12 hours with upLPS; and activated with MSU. NLRP3 was immunoprecipitated and analyzed for pTyr and PTPN22. All data are representative of 1 of 3-5 independent experiments. Numbers below the blots show results of densitometry.

tion increased to almost 100\% (Figure 5E). In cells expressing the autoimmunity-associated PTPN22 variant, NLRP3 phosphorylation was reduced already in the basal state and completely absent upon MSU treatment (Figure 5E), indicating a gain of function of this variant with respect to NLRP3 dephosphorylation.

These data indicate that PTPN22 regulates the activity of the NLRP3 inflammasome by interfering with NLRP3 tyrosine phosphorylation. NLRP3 is thereby phosphorylated at the basal state, and activation is accompanied by decreased phosphory- lation. When PTPN22 is missing, NLRP3 phosphorylation, however, increases upon its activation. In this way, PTPN22 dephosphorylates NLRP3 promoting sustained activation of the NLRP3 inflammasome. These findings reveal a mechanism regulating the NLRP3 inflammasome.

PTPN22 targets NLRP3 at tyrosine residue Tyr861. To further confirm our findings, we transfected the THP-1 cells with PTPN22, NLRP3, or PTPN22 and NLRP3 knockdown constructs. The NLRP3 pTyr band was enhanced in PTPN22-deficient cells 

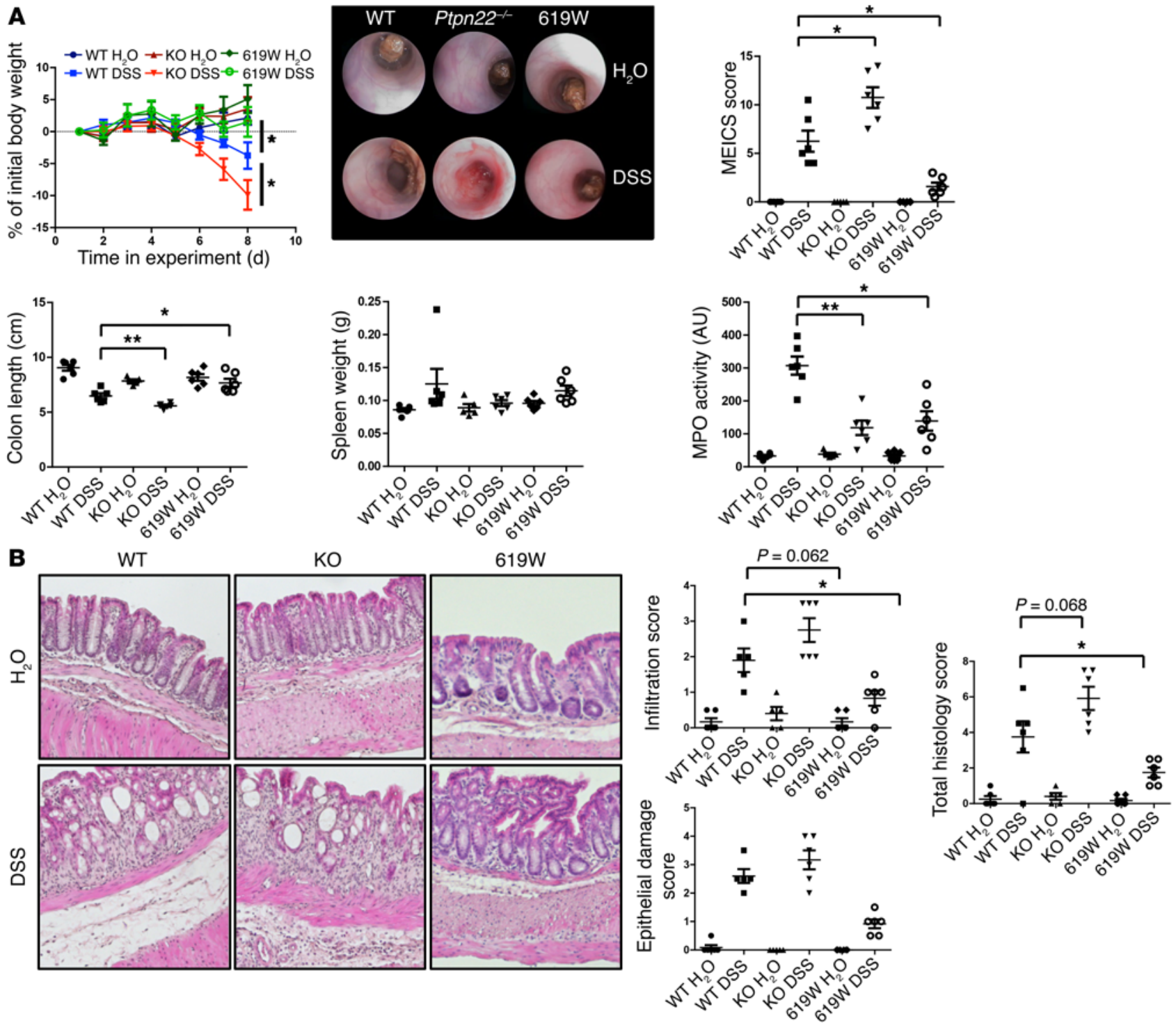

Figure 7. Loss of PTPN22 enhances colitis, while presence of the $619 \mathrm{~W}$ variant is protective. Acute colitis was induced in female WT, Ptpn22 ${ }^{-/}$, or PTPN22-619W (619W) mice by administration of 2.5\% DSS for 7 days. (A) Weight development, representative images from colonoscopy, MEICS score analysis, colon length, spleen weight, and MPO activity. (B) H\&E staining from the distal colon and analysis of epithelial damage and infiltration. All data are representative of 1 of 2 independent experiments with 4-6 mice per group each $(n=4-6)$. Each dot represents one mouse. ${ }^{*} P<0.05$, ${ }^{* *} P<0.01$, Mann-Whitney $U$ test with Bonferroni correction. Original magnification (H\&E), $\times 10$.

but absent in immunoprecipitates from THP-1 cells treated with NLRP3-targeting siRNA (Figure 6A), indicative of the NLRP3 specificity of the pTyr band. To further confirm that PTPN22 directly dephosphorylates NLRP3, we used a cell-free in vitro system (32). We collected phosphorylated NLRP3 from NLRP3-transfected HEK293T cells and PTPN22 from THP-1 cells by immunoprecipitation. As controls, we used those immunoprecipitates from THP-1 cells expressing PTPN22 shRNA in which we did not detect PTPN22; and immunoprecipitates from HEK cells expressing either the autoimmunity-associated variant $(620 \mathrm{~W})$, a loss-offunction PTPN22 variant (263Q), or the related phosphatase PTPN2 (Figure 6B). In the 263Q variant, a crucial arginine in the catalytic domain is mutated to a $\mathrm{Q}$ residue, resulting in a complete loss of phosphatase activity (33). In NLRP3 samples incubated with control immunoprecipitates, we found readily detectable tyrosine phosphorylation, which was not changed upon coincubation with PTPN2 or with loss-of-function PTPN22-263Q. In NLRP3 samples incubated with PTPN22-containing precipitates, however, tyrosine phosphorylation was strongly reduced after 60 minutes (Figure 6B). Consistent with a gain of function, incubation with PTPN22-620W resulted in faster and more efficient NLRP3 dephosphorylation (Figure 6B).

We then studied which tyrosine residue within the NLRP3 protein might be targeted by PTPN22. In humans, NLRP3 exists in several different splice variants, including a full-length $(130-\mathrm{kDa})$ form and an intermediate $(120-\mathrm{kDa})$ form that lacks amino acids 720-776 and 834-890 (34). Interestingly, only one tyrosine residue is located within this sequence at position 861 . Of note, this residue is conserved among mammals (Figure 6C). As we only detected tyrosine phosphorylation in the full-length but not in the 
A
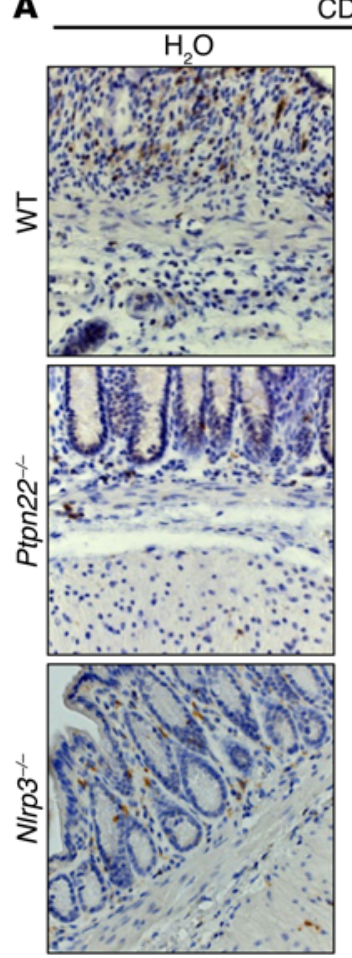

$\mathrm{CD} 3$
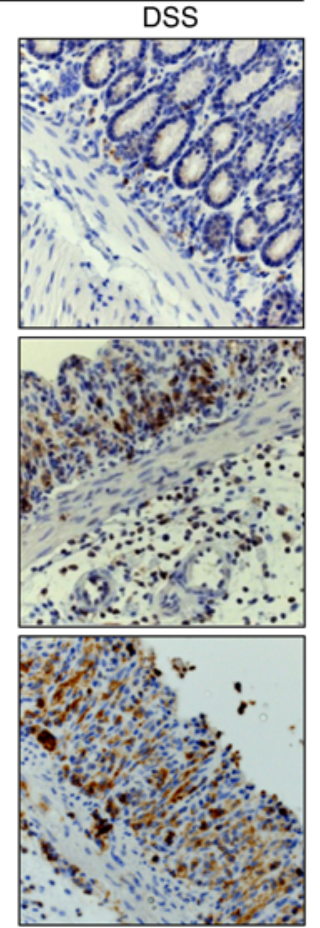

B
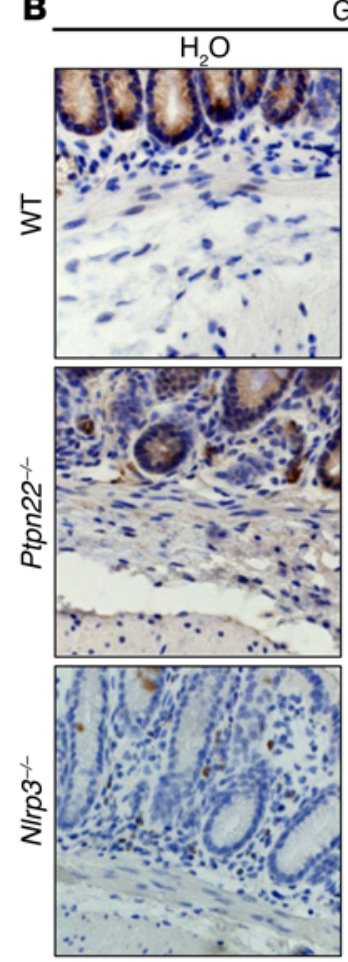

Gr1
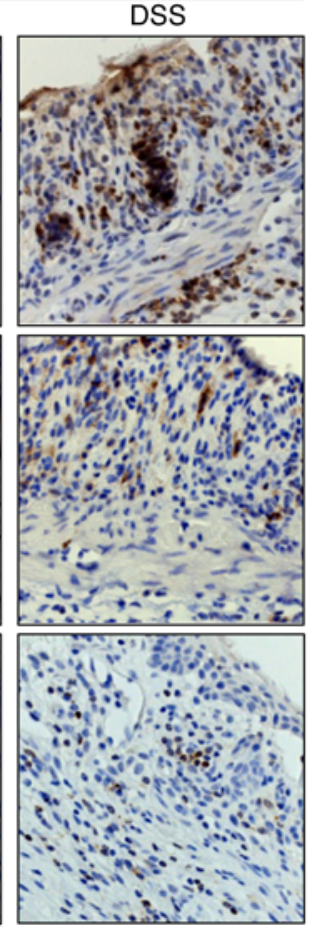

C

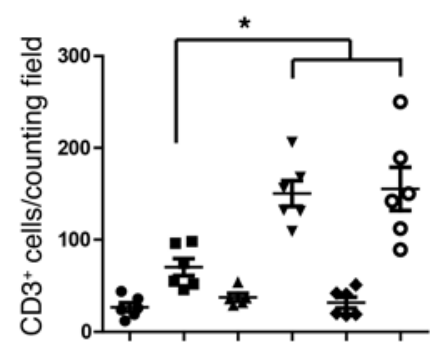

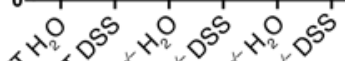
20



Figure 8. Reduced infiltration of granulocytes in the inflamed colon of Ptpn22-/- and $\mathbf{N I r p 3}^{-/-}$mice. Acute colitis was induced in female WT, Ptpn22 ${ }^{-/ \text {, }}$ or $\mathrm{Nlrp3}^{-1-}$ mice by administration of $2.5 \%$ DSS for 7 days. Immunohistochemistry of the distal colon stained for CD3+ $\mathrm{T}$ cells $(\mathbf{A})$ and $\mathrm{Gr} 1^{+}$granulocytes (B). Statistical analysis of infiltrating cells is shown in C. Data are representative of 1 of 2 independent experiments with $4-6$ mice per group each $(n=4-6)$. Each dot represents 1 mouse. ${ }^{*} P<0.05$, Mann-Whitney $U$ test with Bonferroni correction. Original magnification (IHC), $\times 10$.

intermediate form of NLRP3, we hypothesized that the phosphorylated residue might be the tyrosine at position 861 (Tyr861). To test this hypothesis, we transfected HEK293T cells with either a WT NLRP3 vector (WT-NLRP3) or a target mutated form, where Tyr861 was replaced with a phenylalanine residue (Y>F-NLRP3). While tyrosine phosphorylation was detected in WT-NLRP3, this band was completely absent in the Y>F-NLRP3 construct (Figure 6D). This confirms our previous findings and indicates that PTPN22 dephosphorylates NLRP3 at Tyr861.

NLRP3 phosphorylation negatively regulates NLRP3 activation. To test the functional consequence of the absence of NLRP3 phosphorylation, we introduced WT-NLRP3, Y>F-NLRP3, Y>E (mimicking constant phosphorylation of Tyr859 [the mouse ortholog of human Tyr861]), or $\mathrm{Y}>\mathrm{A}$ (negative control for $\mathrm{Y}>\mathrm{E}$ ) vectors into BMDCs prior to inflammasome activation. To rule out interaction of the constructs with endogenous NLRP3, we used Nlrp3 $3^{-/}$or Nlrp3 Ptpn22 double-deficient cells for this study. Transfection with WT-NLRP3 resulted in moderate inflammasome activation and IL-1 $\beta$ secretion following MSU treatment, which was reduced in Ptpn22- cells. When we transfected the cells with the $\mathrm{Y}>\mathrm{F}-$ NLRP3 construct, where Tyr859 phosphorylation is not possible, NLRP3 activation was increased as indicated by elevated caspase- 1 and IL-1 $1 \beta$ cleavage, and loss of PTPN22 was no longer able to reduce NLRP3 activation (Figure 6, E and F). Mimicking constant phosphorylation of NLRP3 using the $\mathrm{Y}>\mathrm{E}$ construct, on the other hand, resulted in abrogation of NLRP3 activity, while mutation to alanine ( $\mathrm{Y}>\mathrm{A}$; a control for glutamate substitution) had the same effect as the $\mathrm{Y}>\mathrm{F}$ substitution (Figure 6, E and F). Of note, the presence of the 619W PTPN22 variant resulted in enhanced NLRP3-mediated IL-1 $\beta$ and caspase-1 activation when the WT NLRP3 construct was used; the presence of the 623Q loss-offunction PTPN22 variant had the opposite effect (Supplemental Figure 5), indicating that PTPN22-619W acts as a gain-of-function variant. On the other hand, neither of the variants affected inflammasome activation in the $\mathrm{Y}>\mathrm{F}$ or $\mathrm{Y}>\mathrm{E}$ NLRP3 constructs (Supplemental Figure 5), showing that the effects on NLRP3 are dependent on NLRP3 phosphorylation at Tyr859. This indicates that PTPN22 enhances NLRP3 activation through dephosphorylation of Tyr861/Tyr859. Taken together, these results suggest that phosphorylation of NLRP3 at Tyr861 (Tyr859 in the mouse) negatively regulates NLRP3 activation.

ASC is necessary for PTPN22-NLRP3 interaction. As a next step, we addressed whether a functional inflammasome is necessary for PTPN22-NLRP3 interaction under physiological conditions. Therefore, we differentiated primary BMDCs from caspase-1-, ASC-, or NLRP3-deficient mice; stimulated them with MSU to induce inflammasome assembly; and immunoprecipitated NLRP3 from the respective lysates. Surprisingly, loss of the adaptor molecule ASC abrogated the direct interaction between NLRP3 and PTPN22, and resulted in enhanced levels of NLRP3 phosphorylation in NLRP3 precipitates. In contrast, in NLRP3 immunoprecipitates from caspase-1-deficient BMDCs, there was detectable tyrosine phosphorylation of NLRP3, which disappeared following treatment with MSU, and this was accompanied by an enhanced NLRP3-PTPN22 interaction. This indicates that caspase-1 activation is dispensable for the observed effects of PTPN22 on NLRP3 phosphorylation (Figure 



WT DSS

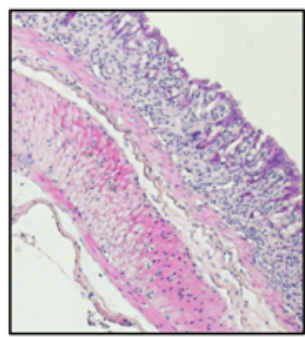

$\mathrm{NIrp3}^{-1-} \mathrm{H}_{2} \mathrm{O}$
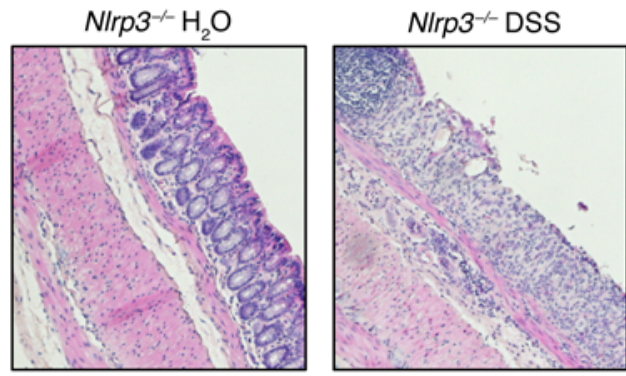

E


6G). Further, we again found that PTPN22-619W constitutively interacted with NLRP3, and this interaction was further enhanced upon MSU stimulation (Figure 6G). Consistently, phosphorylation of NLRP3 was lower in cells expressing PTPN22-619W when compared with cells expressing WT PTPN22 (Figure 6G).

Loss of PTPN22 results in aggravated DSS-mediated intestinal inflammation. To test the in vivo relevance of PTPN22 in intestinal inflammation, we treated WT, Ptpn22-/, and PTPN22-619W mice with DSS for 8 days to induce acute intestinal inflammation. As reported previously (23), loss of PTPN22 resulted in aggravated intestinal inflammation as demonstrated by pronounced weight loss, increased colitis score, and pronounced shortening of the colon, while the presence of PTPN22-619W had a protective effect (Figure 7A). Interestingly, DSS-induced increases in myeloperoxidase (MPO) levels - an indicator of infiltration/activation of neutrophilic granulocytes - which were clearly observable in WT animals, were less pronounced in Ptpn22-- animals (Figure 7A). In H\&E-stained sections from the terminal colon, Ptpn $22^{-/-}$mice harbored increased inflammatory infiltrates upon DSS treatment, while DSS-induced epithelial damage was not altered (Figure 7B). PTPN22-619W mice, on the other hand, showed reduced infiltration and epithelial damage (Figure 7B). While infiltration of $\mathrm{CD}^{+}$cells - indicative of $\mathrm{T}$ lymphocytes - was enhanced in Ptpn $22^{-/}$mice, we found reduced numbers of $\mathrm{Gr}^{+}$cells, indicative of reduced granulocyte infiltration.

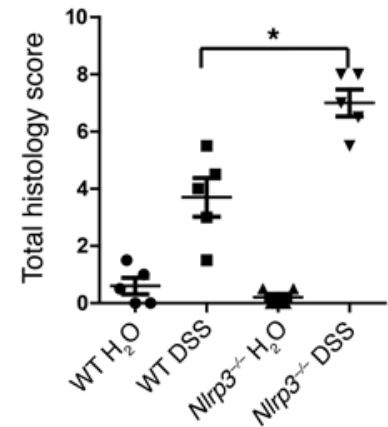

Figure 9. Loss of NLRP3 results in pronounced colitis in our housing conditions. Acute colitis was induced in female WT, Ptpnz2/-, or $\mathrm{Nrp3}^{-/-}$mice by administration of $2.5 \%$ DSS for 7 days. (A) Weight development, (B) MPO activity, and (C) colon length. (D) H\&E staining from the distal colon and (E) analysis of epithelial damage and infiltration. All data are representative of 1 of 2 independent experiments with 4-6 mice per group each $(n=4-6)$. Each dot represents 1 mouse. ${ }^{*} P<0.05,{ }^{*} P<0.01$, Mann-Whitney $U$ test with Bonferroni correction. Original magnification $(\mathrm{H} \& \mathrm{E}$ and $\mathrm{IHC}), \times 10$.
This might explain the observed changes in the MPO assay (Figure 8). Of note, PTPN22-619W mice also show decreased MPO levels when compared with WT animals (Figure 7A), most likely due to overall reduced inflammation and generally reduced infiltration of inflammatory cells into the intestine (Figure 7B). Further, when using NLRP3-deficient ( $\mathrm{Nlrp}^{-/-}$) mice, we observed a phenotype similar to that in Ptpn22-- mice: loss of NLRP3 resulted in aggravated weight loss, enhanced colitis score, pronounced shortening of the colon, and a reduction in MPO levels (Figure 9), accompanied by decreased granulocyte but enhanced $\mathrm{T}$ cell infiltration (Figure 8). Similar results were obtained in bone marrow chimeras, wherein NLRP3 was absent only in in hematopoietic cells but normally expressed in stromal cells (Supplemental Figure 6). This is in line with several previous reports indicating enhanced DSS-induced colitis in $\mathrm{Nlrp3}^{-/-}$mice $(10,35)$. Nevertheless, the colitis phenotype in $N$ lrp $3^{-/-}$mice is controversial $(10,11,35)$.

NLRP3 phosphorylation in the mouse colon is reduced upon DSS treatment. To analyze whether tyrosine phosphorylation of NLRP3 and inflammasome activation play a role in the inflamed intestine, we isolated protein from colon tissue of DSS- and water-treated animals. Western blot analysis showed that DSS administration induced cleavage of caspase- 1 and IL-1 $\beta$, as well as IL-18 cleavage (Figure 10A and Supplemental Figure 7A). We additionally immunoprecipitated NLRP3 from homogenized colon specimens and found basal NLRP3 


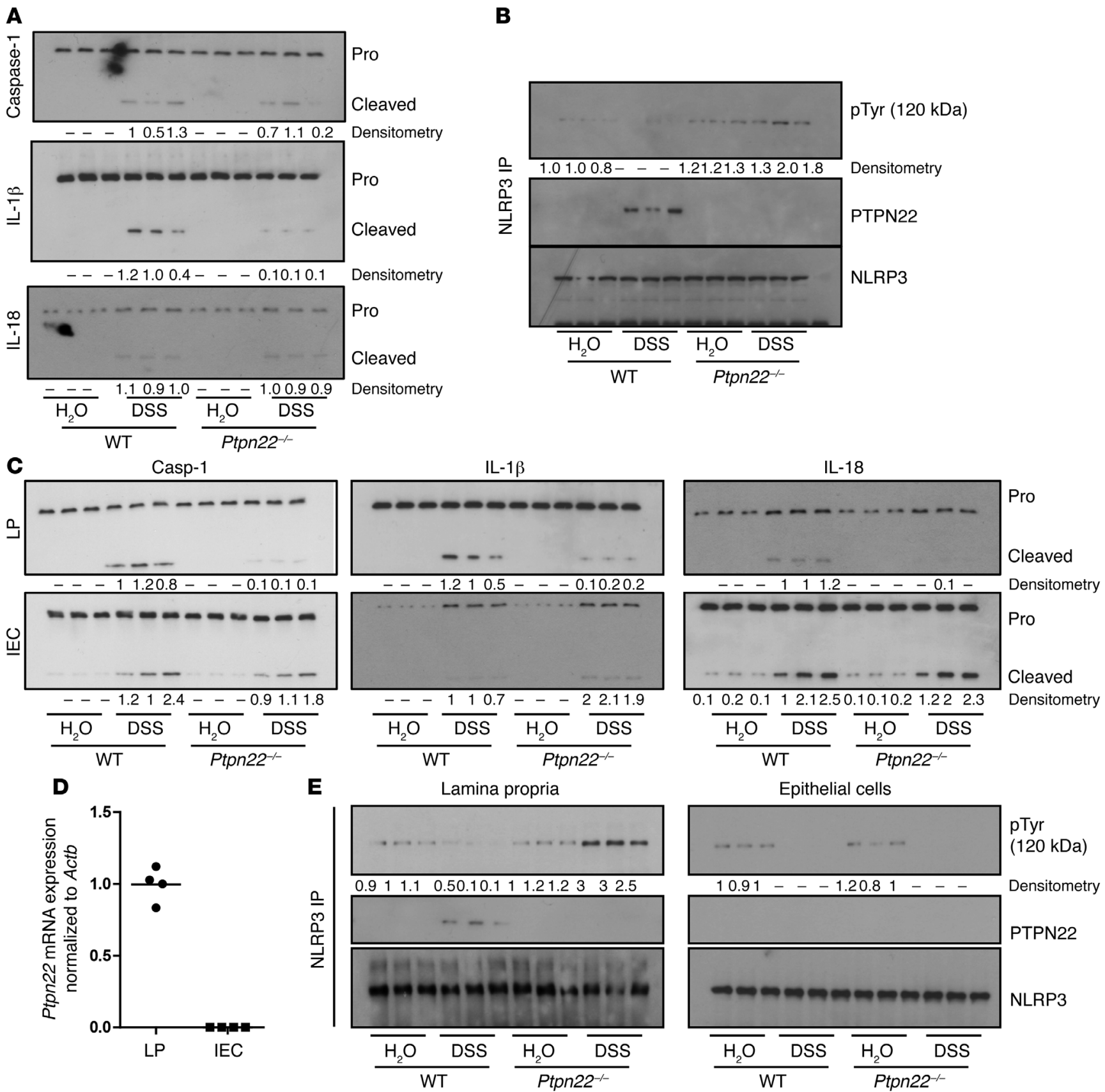

Figure 10. Reduced inflammasome activation in the lamina propria of Ptpn22-/- mice. Colitis was induced in WT and Ptpn22 $2^{-/-}$littermates by administration of $2.5 \%$ DSS for 7 days. (A) Colon specimens were analyzed for caspase-1, IL-1 1 , and IL-18 by Western blot. (B) NLRP3 was immunoprecipitated from whole colon specimens and analyzed for tyrosine phosphorylation and interaction with PTPN22. (C) Intestinal epithelial cell (IEC) fraction and lamina propria (LP) were analyzed for caspase-1, IL-1ß, and IL-18 by Western blot. (D) Lamina propria cells and epithelial cells were analyzed for Ptpn22 mRNA levels normalized to Actb. (E) NLRP3 was precipitated from lamina propria or epithelial cells and analyzed for pTyr and PTPN22. Numbers below the Western blot images show results of densitometry, and each lane represents one mouse.

phosphorylation, which was reduced upon DSS administration (Figure 10B and Supplemental Figure 7A). In samples from Ptpn22-- animals, however, DSS treatment clearly enhanced NLRP3 tyrosine phosphorylation, while the presence of the PTPN22-619W variant further reduced it (Figure 10B and Supplemental Figure 7, A and B).

PTPN22 affects NLRP3 activation only in the lamina propria, but not in epithelial cells. In whole colon specimens from Ptpn22mice, we surprisingly detected only minor changes in DSS-induced caspase-1 or IL-18 activation when compared with WT animals, although IL-1 $\beta$ cleavage was reduced (Figure 10A). In contrast, and consistent with a gain of function of the 619W variant, PTPN22$619 \mathrm{~W}$ animals displayed increased levels of IL- $1 \beta$ and caspase- 1 cleavage (Supplemental Figure 7C). To address in greater detail which compartments of the intestinal wall are responsible for caspase-1 cleavage and IL-1 $1 / \mathrm{IL}-18$ activation, we separated epithelial cells and lamina propria to study inflammasome activation in these two fractions separately. Interestingly, caspase-1, IL-1 1 , and IL-18 cleavage was reduced in the lamina propria but not in the epithelial 



Figure 11. Reduced peritonitis in Ptpn22-/- mice. Acute peritonitis was induced in WT and Ptpn22mice by injection of $1.8 \mathrm{mg}$ MSU for 6 hours. (A) Representative images of flow cytometry, and quantitation of infiltrating neutrophils (B) and T cells (C), as well as IL-1 $\beta$ levels (D) in peritoneal lavages are shown. ${ }^{*} P<0.05,{ }^{* *} P<0.01$ ${ }^{* * *} P<0.001$, Mann-Whitney $U$ test. Data are representative of 1 of 3 independent experiments with 3-5 mice/group.
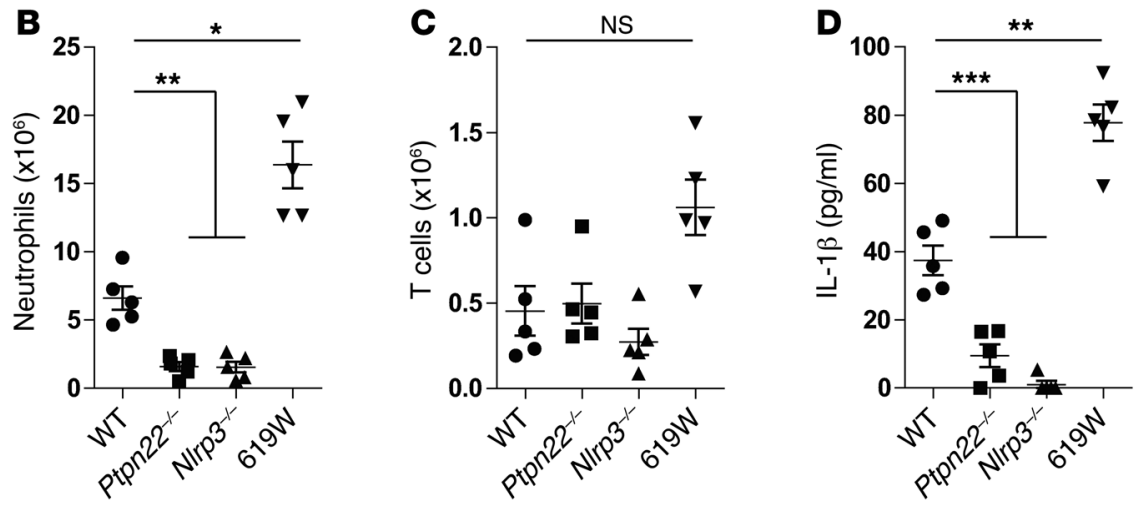

cell compartment in Ptpn22-/- mice (Figure 10C and Supplemental Figure 7D). Further, we found Ptpn 22 mRNA expression only in the lamina propria, but not in the epithelial cell fraction (Figure 10D). In the inflamed intestine, intestinal epithelial cells are the main producers of IL-18, which might explain why overall IL-18 secretion remained unchanged in inflamed colon tissue from $\mathrm{Ptpn} 22^{--}$mice.

As a next step we addressed NLRP3 tyrosine phosphorylation within the intestinal epithelial cell compartment and the lamina propria. In WT animals, we found basal NLRP3 tyrosine phosphorylation in both compartments. Consistent with our finding in the whole colon, DSS treatment reduced NLRP3 phosphorylation in the lamina propria and epithelial cells in WT mice (Figure 10E). In Ptpn $22^{-1-}$ mice, DSS challenge increased NLRP3 phosphorylation in the lamina propria but not in the epithelial cell fraction, where DSS treatment resulted in reduced NLRP3 phosphorylation to a level similar to that in WT animals (Figure 10E).

Loss of PTPN22 reduces $I L-1 \beta$-mediated peritonitis. To further test the physiological in vivo relevance of PTPN22-mediated reduction of IL-1 $\beta$ secretion, we induced MSU-mediated peritonitis in WT, Ptpn22-/- PTPN22-619W, and $\mathrm{Nlrp3}^{-/-}$mice. In this model, IL-1 $\beta$ plays a predominant role in the recruitment of neutrophils (36). Consistent with a reduction in NLRP3-mediated inflammasome activation, peritoneal IL-1 $\beta$ levels, as well as recruitment of neutrophils (but not of $\mathrm{T}$ cells), were reduced in Ptpn22-/ animals, while they were increased in mice expressing the PTPN22-619W variant (Figure 11).

Increased $I L-1 \beta$ secretion in IBD patients carrying an alteredfunction variant of PTPN22. Knockdown or knockout approaches only partially reflect the functional impact of disease-associated variants in the human setting. As a next step, we therefore assessed the impact of the autoimmunity-associated PTPN22 variant rs2476601 (12) in inflammasome activation. To this end, we used intestinal biopsies and serum from CD patients either homozygous for the major $(G)$, heterozygous, or homozygous for the minor (A) PTPN22 allele. Of note, the minor (A) variant results in an altered-function protein product, enhancing PTPN22's in vitro dephosphorylation capacity (17). The presence of rs2476601 confers protection from CD (12) but increases the risk for other autoimmune diseases $(14,15)$. Since the A allele is rare, specimens from only three patients with the AA genotype were available, and intestinal biopsies were obtained from only two of them. The presence of the A allele did not change PTPN22 or NLRP3 mRNA expression (Figure 12A), but both IL1B mRNA expression and secretion of IL- $1 \beta$ were enhanced in carriers of the A allele (Figure 12, A and B). Although the homozygous carriers of the A allele showed the greatest expression of IL1B mRNA, and one of them had extremely high serum IL-1 $\beta$ levels, the number of homozygous patients was far too low to draw conclusions about a possible gene dose effect. In monocyte-derived dendritic cells (mono-DCs) differentiated from the blood of IBD patients heterozygous for the A allele and treated for 6 hours with MSU, we detected increased levels of cleaved caspase- 1 and IL-1 $\beta$ when compared with mono- 
A

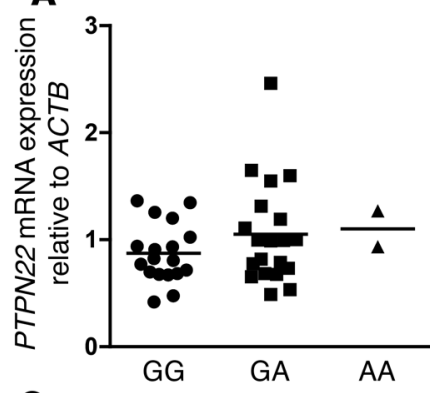

C Healthy controls

IBD patients
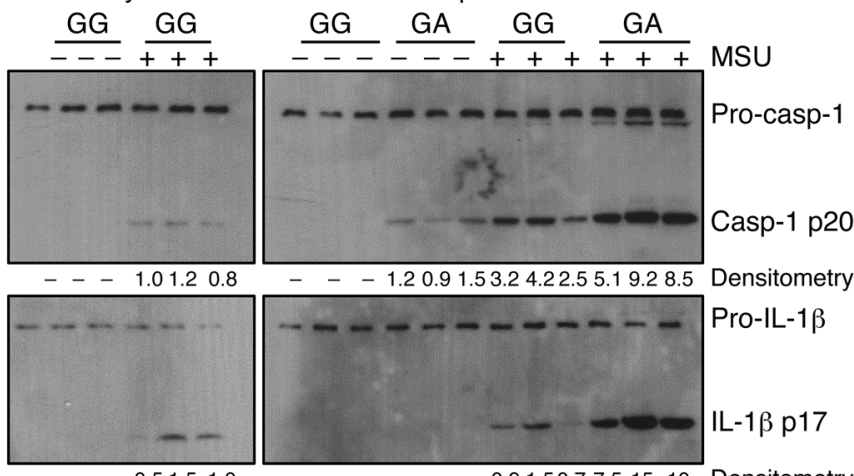

D
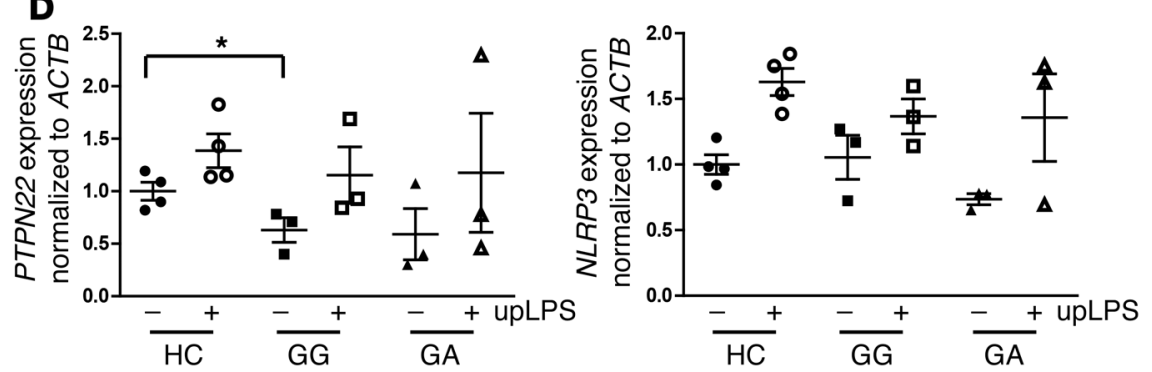
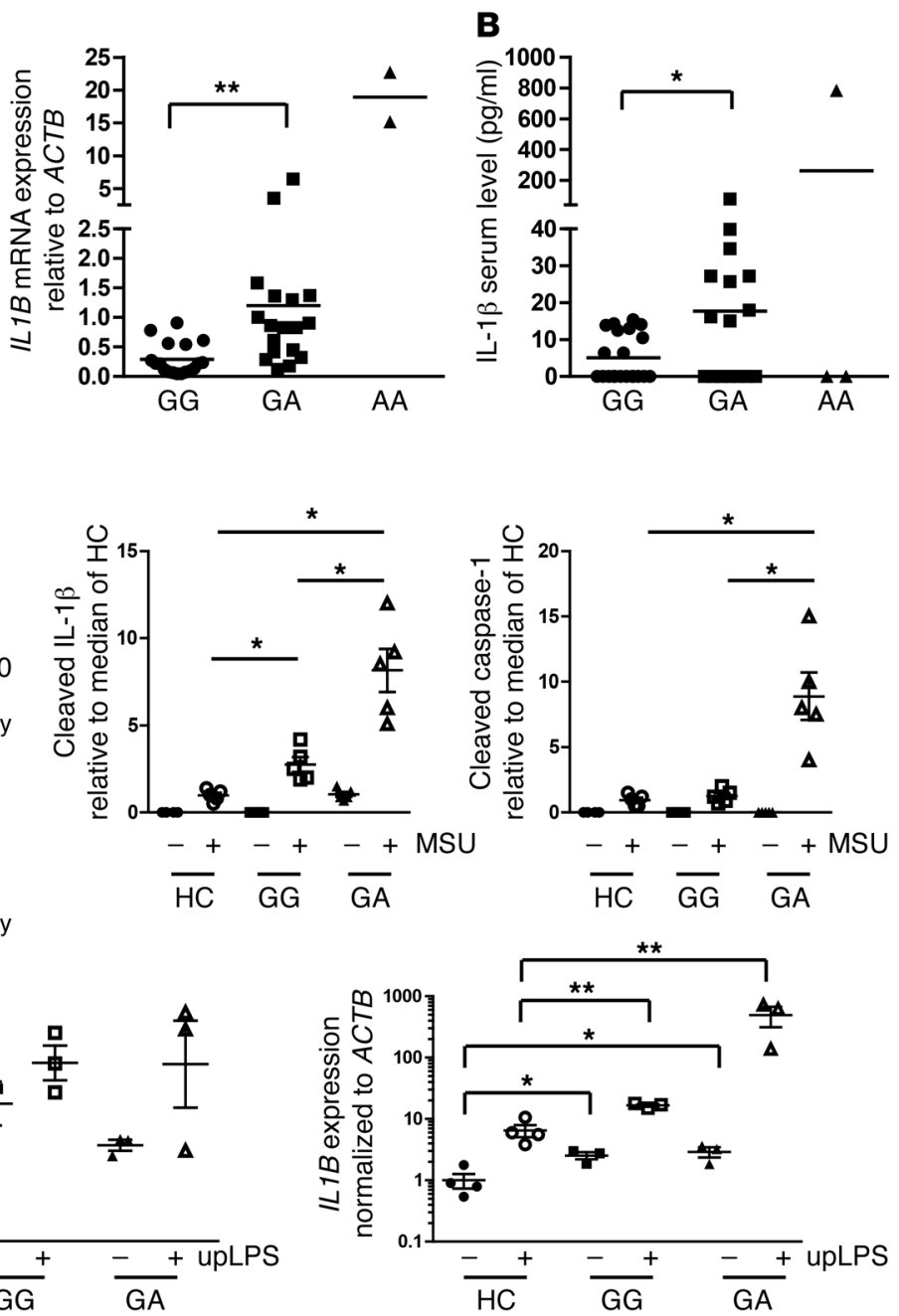

Figure 12. The presence of PTPN22 gain-of-function variant results in increased IL-1 $\beta$ levels. Intestinal biopsies and serum samples from CD patients homozygous for the major (C) variant or heterozygous or homozygous for the minor (A) PTPN22 variant in SNP rs2476601 were analyzed for (A) PTPN22, $N L R P 3$, or IL1B mRNA levels (normalized to $A C T B$ ); and (B) serum levels of IL-1B. Monocyte-derived dendritic cells from healthy controls (HC), CD patients homozygous for the $G$ variant (GC), or CD patients heterozygous for the A variant (GA) were (C) primed for 16 hours with upLPS before activation with MSU for 6 hours prior to analysis for caspase- 1 and IL-1 $\beta$ by Western blot; or (D) left untreated or primed for 16 hours with upLPS before analysis of PTPN22, $N L R P 3$, or ILIB mRNA expression. Data are shown as values relative to nontreated controls and normalized to ACTB. Each dot/lane represents an individual patient; ${ }^{*} P<0.05,{ }^{* *} P<0.001$ (Mann-Whitney $U$ test with Bonferroni correction). Numbers below the Western blot images show results of densitometry.

DCs from IBD patients or healthy controls homozygous for the $G$ allele (Figure 12C). To address whether changes in inflammasome activation might result from changes in LPS-mediated induction of either NLRP3 or IL1B, we further analyzed mRNA expression in upLPS-treated mono-DCs. Mono-DCs from patients heterozygous for the A allele expressed enhanced levels of IL1B mRNA when treated with upLPS, but there was no difference in NLRP3 mRNA expression (Figure 12D). This indicates that increased function of PTPN22 promotes NLRP3 inflammasome activation, likely due to more efficient dephosphorylation. These observations indicate the relevance of our findings for human physiology.

\section{Discussion}

Negative regulation of NLRP3 activity is of crucial importance to control inflammatory responses. However, physiological inflammasome activation is needed for an effective host response and induction of repair mechanisms upon cellular injury and inflam- matory insults (10). Here we describe a mechanism by which the NLRP3 inflammasome is regulated via tyrosine phosphorylation on Tyr861, a process controlled by PTPN22. Interestingly, a naturally occurring NLRP3 variant resulting in a substitution of Tyr861 by a Cys residue causes chronic infantile neurologic cutaneous and articular syndrome (CINCA) (37), a severe form of spontaneous inflammatory disease. This is consistent with our findings using an NLRP3 construct in which Tyr861 is replaced with a phenylalanine residue, which resulted in enhanced NLRP3 activity. Loss of PTPN22 and subsequently increased phosphorylation of NLRP3 at Tyr861, on the other hand, resulted in decreased NLRP3 activation and reduced IL-1 $\beta$ secretion. We demonstrate that PTPN22 directly interacts with NLRP3 and is able to dephosphorylate NLRP3. In a mouse model of colitis as well as in tissue and serum samples from CD patients, we confirmed our in vitro findings and demonstrated the in vivo relevance of our observations. 
The altered-function variant in PTPN22 is associated with enhanced susceptibility to inflammatory diseases, including RA, T1D, and SLE (13-15), where increased levels of IL-1 $\beta$ are observed (16). Of interest, the same variant protects from CD (12). In the literature, conflicting data exist on whether the autoimmunity-associated PTPN22 variant results in enhanced or decreased PTPN22 function. While in vitro data showed that the variant results in enhanced phosphatase activity (17), in vivo data indicated that the variant has effects similar to those of the knockout in terms of $\mathrm{T}$ cell regulation $(25,38,39)$. This discrepancy has been explained by altered substrate specificity of the 620W PTPN22 variant. From our data, we conclude that the autoimmunity-associated variant results clearly in a gain of function in terms of NLRP3 activation. Since we found slightly increased interaction of PTPN22-619W with NLRP3, we conclude that the effects observed here might be the result of both enhanced phosphatase activity and increased substrate interaction. In terms of type I IFN expression and IL-6 secretion, however, the presence of the $619 \mathrm{~W}$ variant results in a loss-of-function phenotype. Since the $619 \mathrm{~W}$ variant in PTPN22 has been suggested to affect substrate specificity or substrate binding capacity (25), we believe that the gain-of-function phenotype regarding NLRP3 activation and the concomitant loss-of-function phenotype regarding type I IFN responses might reflect shifted PTPN22 binding to the respective targets (i.e., equal or slightly increased binding capacity to NLRP3 but reduced binding capacity to molecules involved in type I IFN responses; ref. 25). Given the role described here for PTPN22 in facilitating inflammasome activation, the controversial effect on disease susceptibility in intestinal inflammation and other inflammatory disorders can be explained when considering that NLRP3 activation is involved in wound healing and host defense against invading bacteria in the intestine. In mouse colitis, myeloid cell-derived IL-1 $\beta$ prevents epithelial disruption and promotes wound healing and repair (40). In the more sterile settings of RA and SLE, however, exacerbated NLRP3 activity that might - at least in part - result from increased PTPN22 activity in variant carriers leads to enhanced inflammation. On this background, it is obvious that Ptpn22- animals react less to MSU-induced peritonitis, while they experience aggravated colitis: in the intestine, inflammasome activation is important to protect against invading pathogens and to prevent epithelial disruption/induce epithelial reconstitution (40), and reduction of inflammasome activity results in enhanced colitis severity $(10,11,35)$. Nevertheless, there is controversy regarding the role of NLRP3 in intestinal inflammation, with some reports showing that loss of NLRP3 and subsequent impaired inflammasome activation can protect from colitis (9). This suggests that the role of the NLRP3 inflammasome in colitis might depend on environmental factors. In the setting of sterile MSU-induced peritonitis, inflammasome activation is undoubtedly the main driver of inflammation (36); therefore, its reduction - as observed in Ptpn $22^{-/-}$mice - results in decreased pathology. Since we have shown that the presence of the autoimmunity-associated PTPN22 variant results in increased expression and secretion of IL-1 $\beta$ in CD patients, our data are of relevance for the setting of inflammatory diseases not only in mouse models, but also in patients.

Although PTPN22 was able to dephosphorylate NLRP3 directly in a cell-free system without assembly of the whole inflammasome complex, the interaction of PTPN22 with NLRP3 was dependent on the presence of the inflammasome adaptor ASC within cells. This might be due to the more complex situation within the cell, where ASC might be necessary to stabilize the interaction between NLRP3 and PTPN22. As we found that PTPN22 more efficiently interacts with NLRP3 upon inflammasome induction, ASC might play a role in promoting or stabilizing NLRP3-PTPN22 interaction and subsequent reduction of NLRP3 activity. This would also explain why inflammasome activation is not fully abrogated upon loss of PTPN22. This critical role for ASC in controlling inflammasome activity is in line with a previous report from Hara et al. that showed that ASC phosphorylation is important for controlling inflammasome assembly (4).

While NLRP3 tyrosine phosphorylation is abrogated upon inflammasome activation in PTPN22-competent cells, it is enhanced in response to inflammasome activation in PTPN22-deficient cells. NLRP3 phosphorylation is likely induced by a so-far-unknown kinase that inhibits aberrant inflammasome activity. If PTPN22 - or, in the case of cells that do not express PTPN22, another protein tyrosine phosphatase - is sufficiently activated, NLRP3 tyrosine phosphorylation is limited, resulting in sustained NLRP3 activation.

Several additional mechanisms, including autophagy and noncanonical activation of NLRP3, have been implicated in controlling IL-1 $\beta$ secretion. PTPN22 has been described to affect LPS-induced responses (27), as well as type I IFN responses (28). Both mechanisms are reported to influence caspase- 11 activation, which in turn mediates noncanonical IL-1 $\beta$ secretion/NLRP3 activation (30). Since we did not detect changes in caspase-11 activity upon loss of PTPN22, we assume that noncanonical inflammasome activation is not affected by PTPN22. Autophagy limits IL-1 $\beta$ secretion by targeting pro-IL-1 $\beta$ for degradation (41). However, the function of autophagy in IL-1 $\beta$ secretion is controversial, as a study by Dupont et al. demonstrated a role for autophagy in unconventional protein secretion of IL-1 $\beta$ (42). We have previously shown that loss of PTPN22 results in enhanced autophagy (22); hence, we cannot exclude that loss of PTPN22 additionally affects IL-1 $\beta$ secretion via an indirect mechanism.

Although we show that PTPN22 is necessary for efficient NLRP3 activation in hematopoietic cells, some cell types, including intestinal epithelial cells (43) and fibroblasts (44), express NLRP3 and show efficient NLRP3 activation, although PTPN22 expression is low or even absent in these cells (45). Therefore, we speculate that other phosphatases might also be able to dephosphorylate NLRP3 in these cell types.

In line with previous reports (23), PTPN22-deficient animals experienced aggravated colitis, while PTPN2-619W mice showed the opposite phenotype. Interestingly, levels of granulocyte infiltration and MPO activity were reduced in Ptpn $22^{-/-}$and $\mathrm{Nlpp}^{-/-}$ mice, while all other addressed colitis parameters were enhanced or unchanged. As IL-1 $\beta$ is important for the attraction of granulocytes into the inflamed colon (46), this is consistent with the decreased IL-1 $\beta$ levels observed in the lamina propria of Ptpn $22^{-/-}$ mice. Since MPO not only plays a role in tissue destruction, but is also involved in defense against intracellular bacteria and in limiting T cell-mediated immunity (47), this reduction might actually contribute to disease progression in Ptpn $22^{-/-}$animals. Further, the limiting role of MPO on T cell biology might explain the increased 
number of $\mathrm{CD}^{+}$lymphocytes observed in Ptpn $22^{-/-}$animals. However, since PTPN22 is involved in T cell biology (39), increased numbers of $\mathrm{CD}^{+}$cells might well result from a $\mathrm{T}$ cell-intrinsic effect. Interestingly, the presence of the autoimmunity-associated variant also resulted in decreased MPO levels. However, this was likely due to overall reduced infiltration and not a defect in granulocyte infiltration in response to inflammation.

In summary, we here describe a regulatory mechanism for NLRP3, whereby tyrosine phosphorylation counteracts excessive NLRP3 activation. It has been described that other inflammasome components, such as ASC and NLRC4, are phosphorylated upon inflammasome activation $(4,5)$, suggesting that there are additional mechanisms involved in enhancing inflammasome activity. NLRP3 phosphorylation, in contrast, did not result in activation of the inflammasome complex but decreased the activity of the receptor. Tyrosine phosphorylation can be activating or inhibitory, depending on the assessed molecule and the context, which might explain the opposite effect of tyrosine phosphorylation on different inflammasome components. Hence, NLRP3 phosphorylation is a regulatory mechanism involved in turning off excessive inflammasome activation. As IL- $1 \beta$ is involved in several inflammatory disorders, our findings not only describe what we believe to be a novel mechanism, but potentially have important implications for developing new treatment opportunities.

\section{Methods}

Cells, vectors, and siRNA treatment. THP- 1 and MM6 cells from ATCC were maintained in RPMI-1640 medium (Life Technologies) containing 10\% FCS. To generate BMDCs, bone marrow was flushed from femurs and tibiae of mice and plated in RPMI medium supplemented with $10 \%$ FCS and $30 \%$ conditioned X63-mGMCSF supernatant (X63-mGMCSF cells were generated by our research team). Primary human keratinocytes were isolated, cultured, and activated as described previously (48). PBMCs were collected from whole blood using a Ficoll-Paque (GE Healthcare) density gradient. Knockdown of PTPN22 was induced in THP-1 and MM6 cells using lentiviral particles (Sigma-Aldrich) as described previously (21) or in keratinocytes using three different siRNA constructs (Life Technologies) specifically targeting PTPN22. NLRP3 knockdown was induced using siRNA constructs obtained from Life Technologies as described previously (21). NLRP3 overexpression vectors were constructed by cloning full-length NLRP3 mRNA into a pcDNA3.1-FLAG vector (49). pcDNA3.1 vectors expressing a FLAG-tagged WT human/mouse NLRP3 (NLRP3 WT) or human/mouse NLRP3 with Tyr861>Phe (human) or Tyr859>Phe (mouse) (NLRP3 Y>F), Tyr861/859>Glu (NLRP3 Y>E), and Tyr861/859>Ala (NLRP3 Y $>$ A) mutations, as well as vectors expressing WT, 620/619W, or 263Q PTPN22, were obtained from Geneart. To overexpress WT and mutated NLRP3 vectors in BMDCs, the constructs were subcloned into a pLKO.1 vector (Sigma-Aldrich), and together with pMD2.G and pMCV plasmids (Sigma-Aldrich) used to produce lentiviral particles in HEK293T cells as described previously (21).

Inflammasome activation. THP-1 and MM6 cells were differentiated into macrophages by treatment for 3 hours with $50 \mathrm{nM}$ PMA (Sigma-Aldrich), and all cells were primed for 12 hours with upLPS (Invivogen) prior to inflammasome activation. MSU, ATP, flagellin, and MDP were obtained from Invivogen. MDP was transfected into the cells using FuGENE transfection reagent (Promega). Flagellin and dsDNA were transfected using DOTAP transfection reagent (Roche).
ELISA and LDH release assay. Cell culture supernatants and sera were collected and stored at $-80^{\circ} \mathrm{C}$ until use. ELISA kits detecting human and murine IL-1 $\beta$ were obtained from R\&D Systems; antihuman and anti-mouse IL-18, IL-6, and TNF ELISA kits were obtained from PromoKine; and the LDH release assay was from Roche. Assays were performed according to the manufacturer's instructions using a sample volume of $100 \mu \mathrm{l}$. Absorbance at $450 \mathrm{~nm}$ or $490 \mathrm{~nm}$ in the $\mathrm{LDH}$ release assays, with a correction wavelength of $610 \mathrm{~nm}$, was detected on a SpectraMax M2 fluorescence microplate reader using SoftMax Pro version 5 Software (Molecular Devices). Measurements were performed in duplicate.

Test for biological activity of IL-1 in supernatants. IL-1-responsive D10 G4.1 cells (ATCC) were washed 3 times with assay medium (RPMI 1640 with $10 \%$ FCS and $2.5 \mu \mathrm{g} / \mathrm{ml}$ Concanavalin A; SigmaAldrich) and incubated for 4 hours at $37^{\circ} \mathrm{C}$. Three-fold serial dilutions of recombinant IL- 1 as standard ( $500 \mathrm{pg} / \mathrm{ml}$ in the first wells) or of culture supernatants were prepared in duplicate in 96-well plates. D10 G4.1 cells were then added at $2 \times 10^{4}$ per well to yield a final volume of $100 \mu \mathrm{l}$. After 72 hours of incubation at $37^{\circ} \mathrm{C}$ in humidified air with $5 \%$ $\mathrm{CO}_{2}, 10 \mu \mathrm{l}$ of a mixture of MTS and PMS was added to the wells according to the manufacturer's instructions (Promega). After 2-4 hours the plates were read in an ELISA reader at $490 \mathrm{~nm}$ and evaluated using the SoftMax software. Each 96-well plate contained an IL-1 standard curve. IL-1 specificity of the stimulating activity in the samples was shown by adding the IL-1 receptor antagonist (IL-1Ra, $250 \mathrm{ng} / \mathrm{ml}$; Peprotech) to some of the assayed supernatants. Inhibition was between $40 \%$ and $80 \%$, depending on the amount of IL- 1 in the samples.

Western blotting and immunoprecipitation. Cells or tissue specimens were lysed in M-PER cell lysis buffer (Thermo Fisher Scientific), and immune-reactive proteins were detected using standard Western blot methods. Antibodies used were anti-caspase-3 (Cell Signaling Technologies [CST]; catalog 9662); anti-NLRP3 (Enzo Life Sciences; for mouse: clone Cryo-1, catalog 804-880; for human: Nalpy-3a, 804-881); anti-PTPN22 (Santa Cruz Biotechnology Inc.; T-16, 48922); anti-ASC (CST; E1E3I, 13833); anti-caspase-1 (CST; D7F10, 3866); anti-IL-1ß (CST; 3A6, 12242); AIM2 (Santa Cruz Biotechnology Inc.; D14; sc-137967); NLRC4 (CST; D5Y8E, 12421); PTPN2 (Merck Millipore; CF-41D, PHO3L).

For immunoprecipitation, samples were precleared with Sepharose $\mathrm{G}$ beads (GE Healthcare) and incubated on a rocker overnight at $4^{\circ} \mathrm{C}$ with $10 \mu \mathrm{g} / \mathrm{ml}$ mouse anti-NLRP3 (Enzo Life Sciences) and $10 \mu \mathrm{g} / \mathrm{ml}$ goat anti-PTPN22 (Santa Cruz Biotechnology Inc.; T-16, 48922), prior to precipitation with Sepharose G beads. After washing, the pellet was resuspended in $1 \times$ loading buffer and boiled for $10 \mathrm{~min}-$ utes at $95^{\circ} \mathrm{C}$, and the supernatants were loaded on the gel. For NLRP3 and pTyr detection in immunoprecipitates, mouse anti-NLRP3 (Enzo Life Sciences; Cryo-1, 804-880 or Nalpy-3a, 804-881) and mouse anti-pTyr (CST; P-Tyr-100, 9411) antibodies were used. For detection of PTPN22, rabbit anti-PTPN22 antibody (Santa Cruz Biotechnology Inc.; H-253, sc-134782) was used.

RNA isolation, RT-PCR, and quantitative PCR. Total RNA was isolated using an RNeasy Plus Mini Kit (QIAGEN), and DNA removed by a TURBO DNA-free Kit (Ambion) according to the manufacturer's instructions. RNA concentration was assessed by absorbance at 260 and $280 \mathrm{~nm}$. Complementary DNA (cDNA) synthesis was performed using a High-Capacity cDNA Reverse Transcription Kit (Applied Biosystems) following the manufacturer's instructions. Real-time PCR 
was performed using FAST qPCR Master Mix for Taqman Assays (Applied Biosystems) on a Fast 7900HT Real-Time PCR system using SDS Software (Applied Biosystems). Measurements were performed in triplicate, human $\beta$-actin was used as endogenous control, and results were analyzed by the $\Delta \Delta \mathrm{CT}$ method. The real-time PCR contained an initial enzyme activation step $\left(5\right.$ minutes, $\left.95^{\circ} \mathrm{C}\right)$, followed by 45 cycles consisting of a denaturing $\left(95^{\circ} \mathrm{C}, 15\right.$ seconds $)$ and an annealing/extending $\left(60^{\circ} \mathrm{C}, 1\right.$ minute) step. The gene expression assays used were all obtained from Life Technologies.

In vitro dephosphorylation assay. For in vitro dephosphorylation studies, NLRP3 vector was transfected into HEK293T cells and isolated using anti-FLAG gel (Invitrogen). NLRP3 was then incubated as described previously $(32)$ at $30^{\circ} \mathrm{C}$ in phosphatase buffer $(25 \mathrm{mM}$ Tris pH 7.5, $5 \mathrm{mM}$ DTT, $1 \mathrm{mg} / \mathrm{ml} \mathrm{BSA}, 2.5 \mathrm{mM}$ EDTA) for 60 minutes in the presence or absence of PTPN22 immunoprecipitated from THP-1 cells.

Mouse strains, housing, induction of colitis, and peritonitis. PTPN22-deficient (Ptpn22--) mice in a C57BL/6 background were obtained from Genentech (50) and bred with pure C57BL/6 mice to generate Ptpn $22^{+/-}$mice. Ptpn $22^{+/-}$mice were then crossed with each other to obtain Ptpn $22^{-/-}$and Ptpn $22^{+/+}$littermates. Mice expressing the 619W variant in PTPN22 (PTPN22-619W mice) in a C57BL/6 background were were generated in our laboratory (25) and backcrossed with pure C57BL/6 mice to generate heterozygous mice. These mice were then crossed with each other to obtain PTPN22-619W and PTPN22-WT littermates. Ten- to 12-week-old female littermates were used for all studies. Mice were kept in a specific pathogen-free (SPF) facility. Acute colitis was induced by administration of 2.5\% DSS (MP Biomedicals) in the drinking water for 7 days. Colonoscopy was performed as described previously and scored using the murine endoscopic index of colitis severity (MEICS) scoring system (51). Histological scoring for inflammatory infiltration and epithelial cell damage was performed on H\&E-stained section of the most distal $1 \mathrm{~cm}$ of the mouse colon as described previously $(51,52)$. Peritonitis was induced as described previously (36). In brief, mice were injected with $1.8 \mathrm{mg}$ MSU for 6 hours and peritoneal lavages analyzed by flow cytometry.

Bone marrow chimeras. Bone marrow chimeras were generated as described previously (53). In brief, recipient mice were irradiated with a single dose of $9.5 \mathrm{~Gy}$. Bone marrow was isolated from donors by flushing femora and tibiae with sterile PBS. Single-cell suspensions were prepared, and $2 \times 10^{6}$ cells were injected intravenously into recipient mice 6 hours after irradiation. Eight weeks after bone marrow transfer, colitis was induced by administration of $1.5 \%$ DSS for 7 days.

MPO activity assay. Colon specimens were homogenized in $50 \mathrm{mM}$ phosphate buffer ( $\mathrm{pH}$ 6.0) and $0.5 \%$ hexadecyltrimethylammonium bromide (Sigma-Aldrich) using a gentleMACS tissue homogenizer (Miltenyi Biotec). After three freeze-and-thaw cycles, supernatant was mixed with $0.02 \%$ dianisidine (Sigma-Aldrich) in $50 \mathrm{mM}$ phosphate buffer, $\mathrm{pH}$ 6.0, and $0.0005 \% \mathrm{H}_{2} \mathrm{O}_{2}$ (Sigma-Aldrich). MPO activity, expressed as arbitrary units, was calculated as mean absorbance (460 $\mathrm{nm}$ ) per incubation time (in minutes) per protein content (in grams).

Immunohistochemistry. Sections from the most distal $1 \mathrm{~cm}$ of the mouse colon were deparaffinized, and antigen was retrieved using citrate buffer, pH 6.0 (Dako). Endogenous peroxidases were inhibited by incubation with $0.9 \%$ hydrogen peroxide for 15 minutes at room temperature (RT), and blocking was performed using 3\% BSA. Sections were incubated with rabbit anti-CD3 (Abcam; ab5690) or rat anti-Ly6G antibody (Biolegend; clone 1A8). HRP-labeled secondary antibody (Santa Cruz Biotechnology Inc.) was then applied for 1 hour at RT and antibody binding visualized by a Liquid DAB+ Substrate Chromogen System (Dako). Samples were counterstained with hematoxylin, incubated in ethanol and xylol solutions of ascending concentrations, and finally mounted. Microscopic assessment was performed using an AxioCam HRc (Zeiss) on a Zeiss Axio Imager.Z2 microscope with AxioVision release 4.8.2 software.

Patient samples. Intestinal tissue specimens were taken by endoscopy from the terminal ileum, colon, or rectum of $\mathrm{CD}$ patients with active or inactive disease. Biopsy samples were taken from regions appearing macroscopically inflamed in patients with active disease and from normal-appearing regions in patients with quiescent disease. Serum samples were collected from a partially overlapping patient group. Patients were genotyped for the presence of the PTPN22 rs2476601 and grouped into homozygous, heterozygous, and non-carriers for the minor (A) allele. The heterozygous and non-carrier groups were matched for age, sex, and disease activity. PBMCs were isolated from fresh blood samples as described previously (21), CD14 ${ }^{+}$cells sorted using magnetic beads (Miltenyi Biotec), and monocyte-derived dendritic cells differentiated for 5 days in RPMI 1640 supplemented with $10 \%$ FCS, 500 U hIL-4, and 1000 U hGM-CSF.

Statistics. Data are presented as mean \pm SEM for a series of $n$ experiments. Data are expressed as relative values of the respective control groups. Statistical analysis was performed using ANOVA followed by Student-Newman-Keuls post hoc test, 2-tailed Student's $t$ test (cell culture experiments), or Mann-Whitney $U$ test (animal experiments and patient samples) where appropriate. $P$ values less than 0.05 were considered significant.

Study approval. For patient data, written informed consent was obtained before specimen collection, and studies were approved by the local Ethics Committee (Ethikkommission des Kantons Zürich, Zurich, Switzerland). Mouse experiments were performed with the approval of the local animal welfare authority (Tierschutzkommision Zürich, Zurich, Switzerland).

\section{Author contributions}

MRS performed experiments, analyzed the data, and wrote the first draft of the manuscript. SK, CG, SL, KA, and WF performed experiments and were involved in data analysis. PMG and MGG were involved in vector design and subcloning. DJR and XD generated PTPN22-619W mice. HDB and EC performed experiments in keratinocytes and were involved in data analysis and interpretation. FM and BB performed experiments. ACC corrected and approved the manuscript. SS, SRV, MF, GR, and MS were involved in acquisition of patient samples. MS and GR conceived, designed, and supervised the study. All authors wrote, corrected, and approved the manuscript.

\section{Acknowledgments}

This research was supported by a grant from Fonds zur Förderung des akademischen Nachwuchses of the Zürcher Universitätsverein to M. Scharl; a research grant from the Swiss Philanthropy Foundation to M. Scharl and G. Rogler; a research credit grant from the University of Zurich to M. Scharl; research grants from the Swiss National Science Foundation to M. Scharl (no. 314730146204 and no. CRSII3_154488/1, subproject 2), G. Rogler (no. 310030-120312), S.R. Vavricka (no. 320000-114009/3 and no. 
32473B_135694/1), and the Swiss IBD Cohort (no. 3347CO108792); a grant from the Zurich Center for Integrative Human Physiology of the University of Zurich to M. Scharl; a grant from the Novartis Foundation for Biomedical Research to M. Scharl; a grant from the Hartmann-Müller Foundation to M. Scharl; and the Abbvie IBD Award 2012 to M. Scharl. The funding institutions had no role in the study design; in the collection, analysis, and inter- pretation of data; in the writing of the manuscript; or in the decision to submit the manuscript for publication.

Address correspondence to: Michael Scharl, Division of Gastroenterology and Hepatology, University Hospital Zurich, Rämistrasse 100, 8091 Zurich, Switzerland. Phone: 41.44.255.9519; E-mail: michael.scharl@usz.ch.
1. Keller M, Ruegg A, Werner S, Beer HD. Active caspase- 1 is a regulator of unconventional protein secretion. Cell. 2008;132(5):818-831.

2. Chen GY, Nunez G. Sterile inflammation: sensing and reacting to damage. Nat Rev Immunol. 2010;10(12):826-837.

3. Martinon F, Mayor A, Tschopp J. The inflammasomes: guardians of the body. Annu Rev Immunol. 2009;27:229-265.

4. Hara H, et al. Phosphorylation of the adaptor ASC acts as a molecular switch that controls the formation of speck-like aggregates and inflammasome activity. Nat Immunol. 2013;14(12):1247-1255.

5. Qu Y, et al. Phosphorylation of NLRC4 is critical for inflammasome activation. Nature. 2012;490(7421):539-542.

6. Villani AC, et al. Common variants in the NLRP3 region contribute to Crohn's disease susceptibility. Nat Genet. 2009;41(1):71-76.

7. Martinon F, Tschopp J. Inflammatory caspases: linking an intracellular innate immune system to autoinflammatory diseases. Cell. 2004;117(5):561-574.

8. Street ME, et al. Relationships between serum IGF-1, IGFBP-2, interleukin-1 $\beta$ and interleukin-6 in inflammatory bowel disease. Horm Res. 2004;61(4):159-164.

9. Bauer C, et al. Colitis induced in mice with dextran sulfate sodium (DSS) is mediated by the NLRP3 inflammasome. Gut. 2010;59(9):1192-1199.

10. Zaki MH, Boyd KL, Vogel P, Kastan MB, Lamkanfi M, Kanneganti TD. The NLRP3 inflammasome protects against loss of epithelial integrity and mortality during experimental colitis. Immunity. 2010;32(3):379-391.

11. Chen GY, Nunez G. Inflammasomes in intestinal inflammation and cancer. Gastroenterology. 2011;141(6):1986-1999.

12. Diaz-Gallo LM, et al. Differential association of two PTPN22 coding variants with Crohn's disease and ulcerative colitis. Inflamm Bowel Dis. 2011;17(11):2287-2294.

13. Begovich AB, et al. A missense single-nucleotide polymorphism in a gene encoding a protein tyrosine phosphatase (PTPN22) is associated with rheumatoid arthritis. Am J Hum Genet. 2004;75(2):330-337.

14. Kyogoku C, et al. Genetic association of the R620W polymorphism of protein tyrosine phosphatase PTPN22 with human SLE. Am J Hum Genet. 2004;75(3):504-507.

15. Bottini N, et al. A functional variant of lymphoid tyrosine phosphatase is associated with type I diabetes. Nat Genet. 2004;36(4):337-338.

16. Yang Q, et al. Deregulated NLRP3 and NLRP1 inflammasomes and their correlations with disease activity in systemic lupus erythematosus. JRheumatol. 2014;41(3):444-452.
17. Vang T, et al. Autoimmune-associated lymphoid tyrosine phosphatase is a gain-of-function variant. Nat Genet. 2005;37(12):1317-1319.

18. Duerr RH, et al. A genome-wide association study identifies IL23R as an inflammatory bowel disease gene. Science. 2006;314(5804):1461-1463.

19. Wellcome Trust Case Control Consortium. Genome-wide association study of 14,000 cases of seven common diseases 3,000 shared controls. Nature. 2007;447(7145):661-678.

20. Lasbleiz S, et al. Linkage proof for PTPN22, a rheumatoid arthritis susceptibility gene a human autoimmunity gene. Proc Natl Acad Sci US A. 2007;104(5):1649-1654.

21. Spalinger MR, et al. Loss of protein tyrosine phosphatase nonreceptor type 22 regulates interferon- $\gamma$-induced signaling in human monocytes. Gastroenterology. 2013;144(5):978-988.e910.

22. Spalinger MR, Lang S, Vavricka SR, Fried M, Rogler G, Scharl M. Protein tyrosine phosphatase non-receptor type 22 modulates NOD2induced cytokine release autophagy. PLoS One. 2013;8(8):e72384.

23. Wang Y, et al. The autoimmunity-associated gene PTPN22 potentiates toll-like receptor-driven, type 1 interferon-dependent immunity. Immunity. 2013;39(1):111-122.

24. Zhang J, et al. The autoimmune disease-associated PTPN22 variant promotes calpain-mediated Lyp/Pep degradation associated with lymphocyte dendritic cell hyperresponsiveness. Nat Genet. 2011;43(9):902-907.

25. Dai X, et al. A disease-associated PTPN22 variant promotes systemic autoimmunity in murine models. JClin Invest. 2013;123(5):2024-2036.

26. Wu C, et al. BioGPS: an extensible and customizable portal for querying and organizing gene annotation resources. Genome Biol. 2009;10(11):R130.

27. Wang Y, et al. PTPN22 variant R620W is associated with reduced toll-like receptor 7-induced yype I interferon in systemic lupus erythematosus. Arthritis Rheumatol. 2015;67(9):2403-2414.

28. Holmes DA, et al. Autoimmunity-associated protein tyrosine phosphatase PEP negatively regulates IFN- $\alpha$ receptor signaling. J Exp Med. 2015;212(7):1081-1093.

29. Rathinam VA, et al. TRIF licenses caspase-11dependent NLRP3 inflammasome activation by gram-negative bacteria. Cell.2012;150(3):606-619.

30. Broz P, et al. Caspase-11 increases susceptibility to Salmonella infection in the absence of caspase-1. Nature. 2012;490(7419):288-291.

31. Hornung V, et al. AIM2 recognizes cytosolic dsDNA forms a caspase-1-activating inflammasome with ASC. Nature. 2009;458(7237):514-518.

32. Meyer T, Marg A, Lemke P, Wiesner B, Vinke- meier U. DNA binding controls inactivation and nuclear accumulation of the transcription factor Stat1. Genes Dev. 2003;17(16):1992-2005.

33. Orru V, et al. A loss-of-function variant of PTPN22 is associated with reduced risk of systemic lupus erythematosus. Hum Mol Genet. 2009;18(3):569-579.

34. Aganna E, et al. Association of mutations in the NALP3/CIAS1/PYPAF1 gene with a broad phenotype including recurrent fever, cold sensitivity, sensorineural deafness, AA amyloidosis. Arthritis Rheum. 2002;46(9):2445-2452.

35. Allen IC, et al. The NLRP3 inflammasome functions as a negative regulator of tumorigenesis during colitis-associated cancer. J Exp Med. 2010;207(5):1045-1056.

36. Chen C-J, et al. MyD88-dependent IL-1 receptor signaling is essential for gouty inflammation stimulated by monosodium urate crystals. J Clin Invest. 2006;116(8):2262-2271.

37. Frenkel J, Van Kempen MJA, Kuis W, Van Amstel HKP. Variant chronic infantile neurologic, cutaneous, articular syndrome due to a mutation within the leucine-rich repeat domain of CIAS1. Arthritis Rheumatol. 2004;50(8):2719-2720.

38. Brownlie RJ, Miosge LA, Vassilakos D, Svensson LM, Cope A, Zamoyska R. Lack of the phosphatase PTPN22 increases adhesion of murine regulatory $\mathrm{T}$ cells to improve their immunosuppressive function. Sci Signal. 2012;5(252):ra87.

39. Maine CJ, et al. PTPN22 alters the development of regulatory T cells in the thymus. J Immunol. 2012;188(11):5267-5275.

40. Bersudsky M, et al. Non-redundant properties of IL-1alpha and IL-1beta during acute colon inflammation in mice. Gut. 2014;63(4):598-609.

41. Saitoh T, et al. Loss of the autophagy protein Atg16L1 enhances endotoxin-induced IL-1[bgr] production. Nature. 2008;456(7219):264-268

42. Dupont N, Jiang S, Pilli M, Ornatowski W, Bhattacharya D, Deretic V. Autophagy-based unconventional secretory pathway for extracellular delivery of IL-1beta. EMBO J. 2011;30(23):4701-4711.

43. Kummer JA, et al. Inflammasome components NALP 1 and 3 show distinct but separate expression profiles in human tissues suggesting a site-specific role in the inflammatory response. J Histochem Cytochem. 2007;55(5):443-452.

44. Artlett CM, Sassi-Gaha S, Rieger JL, Boesteanu AC, Feghali-Bostwick CA, Katsikis PD. The inflammasome activating caspase 1 mediates fibrosis myofibroblast differentiation in systemic sclerosis. Arthritis Rheumatol. 2011;63(11):3563-3574.

45. Wang S, Dong H, Han J, Ho WT, Fu X, Zhao ZJ. Identification of a variant form of tyrosine phosphatase LYP. BMC Mol Biol. 2010;11:78.

46. Coccia M, et al. IL-1beta mediates chronic intestinal inflammation by promoting the accumulation 
of IL-17A secreting innate lymphoid cells CD4(+) Th17 cells. J Exp Med. 2012;209(9):1595-1609.

47. Odobasic D, et al. Neutrophil myeloperoxidase regulates T-cell-driven tissue inflammation in mice by inhibiting dendritic cell function. Blood . 2013;121(20):4195-4204.

48. Sollberger G, Strittmatter GE, Kistowska M, French LE, Beer HD. Caspase- 4 is required for activation of inflammasomes. JImmunol. 2012;188(4):1992-2000.
49. Geertsma ER, Dutzler R. A versatile and efficient high-throughput cloning tool for structural biology. Biochemistry. 2011;50(15):3272-3278.

50. Hasegawa K, Martin F, Huang G, Tumas D, Diehl L, Chan AC. PEST domain-enriched tyrosine phosphatase (PEP) regulation of effector/memory T cells. Science. 2004;303(5658):685-689.

51. Becker C, Fantini MC, Neurath MF. High resolution colonoscopy in live mice. Nat Protoc. 2006;1(6):2900-2904.
52. Obermeier F, Kojouharoff G, Hans W, Scholmerich J, Gross V, Falk W. Interferon-gamma (IFN-gamma)- and tumour necrosis factor (TNF)-induced nitric oxide as toxic effector molecule in chronic dextran sulphate sodium (DSS)-induced colitis in mice. Clin Exp Immunol. 1999;116(2):238-245.

53. Holl EK. Generation of bone marrow and fetal liver chimeric mice. Methods Mol Biol. 2013;1032:315-321. 